\title{
Modelling and analysis of offshore energy hubs
}

\author{
Hongyu Zhanga,*, Asgeir Tomasgard ${ }^{\mathrm{a}}$, Brage Rugstad Knudsen ${ }^{\mathrm{b}}$, Harald G. \\ Svendsen $^{\mathrm{b}}$, Steffen J. Bakker ${ }^{\mathrm{a}}$, Ignacio E. Grossmann ${ }^{\mathrm{c}}$ \\ ${ }^{a}$ Department of Industrial Economics and Technology Management, Norwegian \\ University of Science and Technology, Høgskoleringen 1, 7491, Trondheim, Norway \\ ${ }^{b}$ SINTEF Energy Research, Kolbjørn Hejes vei 1B, 7491, Trondheim, Norway \\ ${ }^{c}$ Department of Chemical Engineering, Carnegie Mellon University, 5000 Forbes Avenue, \\ Pittsburgh, PA 15213, USA
}

\begin{abstract}
Clean, multi-carrier Offshore Energy Hubs (OEHs) may become pivotal for efficient offshore wind power generation and distribution. In addition, OEHs may provide decarbonised energy supply for maritime transport, oil and gas recovery, and offshore farming while also enabling conversion and temporary storage of liquefied decarbonised energy carriers for export. Here, we investigate the role of OEHs in the transition of the Norwegian continental shelf energy system towards zero-emission energy supply. We develop a mixedinteger linear programming model for investment planning and operational optimisation to achieve decarbonisation at minimum costs. We consider clean technologies, including offshore wind, offshore solar, OEHs and subsea cables. We conduct sensitivity analysis on $\mathrm{CO}_{2}$ tax, $\mathrm{CO}_{2}$ budget and the capacity of power from shore. The results show that (a) a hard carbon cap is necessary for stimulating a zero-emission offshore energy system; (b) offshore wind integration and power from shore can more than halve current emissions, but OEHs with storage are necessary for zero-emission production and (c) at certain $\mathrm{CO}_{2}$ tax levels, the system with OEHs can potentially reduce $\mathrm{CO}_{2}$ emissions by $50 \%$ and energy losses by $10 \%$, compared to a system with only offshore renewables, gas turbines and power from shore.
\end{abstract}

Keywords: clean offshore energy hub, sensitivity analysis, deterministic

Abbreviations: NCS, Norwegian continental shelf; OEH, Offshore energy hub; PFS, Power from shore; Base, The case with only offshore renewables, gas turbines and power from shore; S1, Scenario 1; S2, Scenario 2; MILP, Mixed-integer linear programming.

*Corresponding author's email address: hongyu.zhang@ntnu.no 
mixed-integer linear programming model

\section{Introduction}

In 2020, the European Green Deal lifted the EU climate target aiming to reduce greenhouse gas emissions by at least $55 \%$ by 2030 and become the world's first carbon-neutral continent by 2050 [10]. The North Sea region may play a central role in this with a capacity target of $300 \mathrm{GW}$ for offshore wind power by 2050 [9]. Offshore Energy Hubs (OEHs) and the hub-and-spoke concept offer a transnational and cross-sector solution for better harnessing offshore wind and integration with the rest of the energy system [34]. An energy hub is a physical energy connection point with energy storage where multiple energy carriers can be converted, conditioned [13].

Using OEHs to effectively exploit offshore wind power to decarbonise the Norwegian Continental Shelf (NCS) energy system may contribute to meeting Norway's and Europe's climate targets. Norway was the world's thirdlargest exporter of natural gas in 2019 [24]. Offshore oil and gas extraction was responsible for $26.6 \%$ (13.3 $\mathrm{Mt} \mathrm{CO}_{2}$ equivalent) of the total Norwegian greenhouse gases in 2020 [45]. Norway steps up its climate goal to reduce emissions by $50 \%$ - $55 \%$ by 2030 compared to 1990 levels [29].

This paper investigates the role of clean OEHs in reducing $\mathrm{CO}_{2}$ emissions from the NCS with a focus on NCS oil and gas fields. Figure 1 visualises a potential NCS energy system with OEHs, while Figure 2 illustrates the functioning of an $\mathrm{OEH}$. We only consider producing green hydrogen from electrolysis. A likely first use of OEHs may be to provide decarbonised energy supply for offshore oil and gas recovery, maritime cargo transport, and offshore farming [28]. In the longer term, the development of OEHs may also facilitate the transition to decarbonised continental energy export. After the lifetimes of oil and gas fields, OEHs may produce and export green hydrogen and connect with a North Sea offshore grid [43, 15, 34, 5].

We develop a multi-carrier Mixed-Integer Linear Programming (MILP) model for investment planning optimisation with a high degree of operational details to cope with the short-term characteristics of the problem. We consider investment in clean technologies, including offshore wind, offshore solar, OEHs and Power From Shore (PFS). Offshore oil and gas platforms involve multiple energy carriers, including electricity, heat, gas, oil and water. More potential energy carriers such as hydrogen and ammonia may become critical 


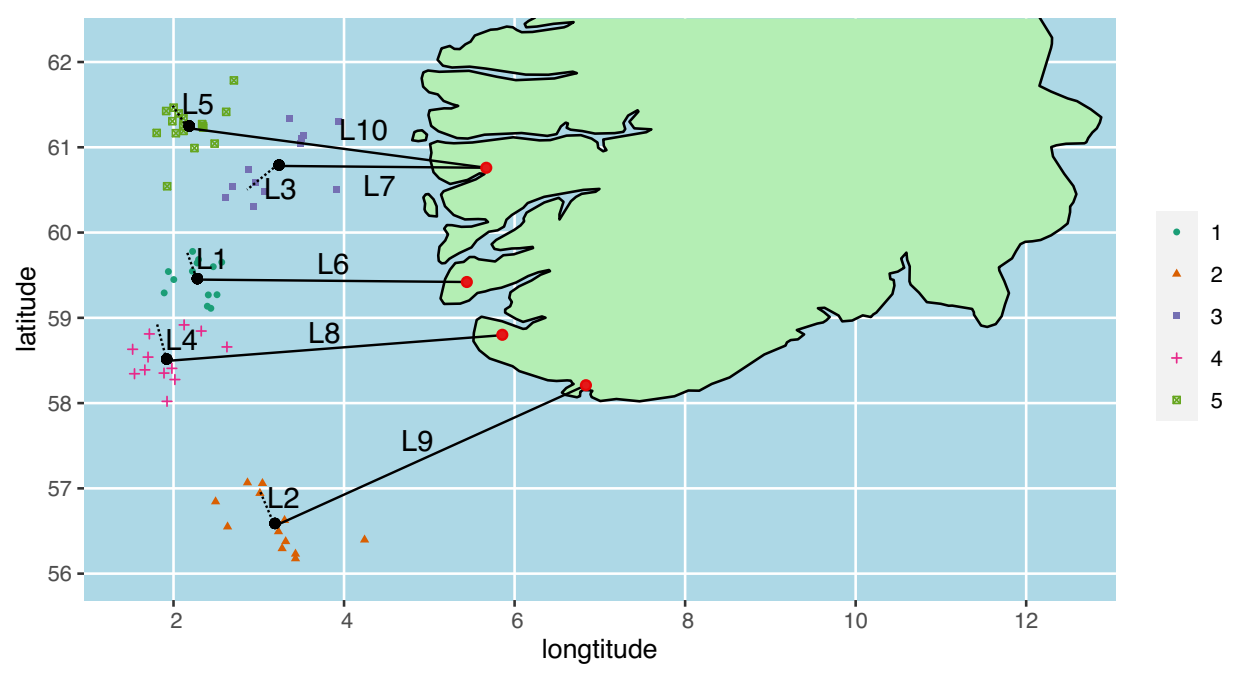

Figure 1: Illustration of the NCS energy system with energy hubs. L1 - L5 (dotted lines) are representative HVAC cables, while L6 - L10 (solid lines) are HVDC cables. Black dots represent energy hubs and the red dots represent the onshore buses they connect to. Points with different shapes and colours represent NCS oil and gas fields.

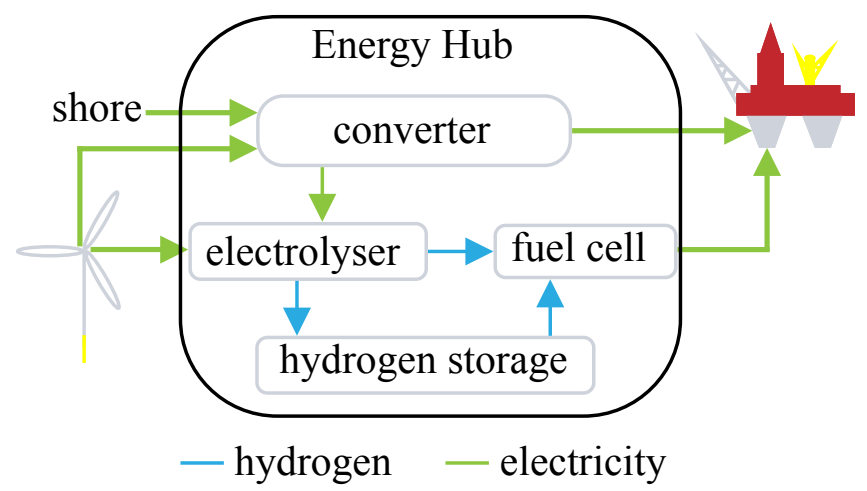

Figure 2: Conceptual illustration of OEHs.

during the offshore energy system transition [12]. The operational model has an hourly time resolution. It is crucial to consider hourly operational decisions in a system with higher penetration of intermittent renewable energy to systematically assess the trade-off between long-term investment decisions and short-term operational decisions [22]. This is even more critical to be an acceptable alternative to current fossil-based energy provision for instal- 
lations on the NCS where the security of supply is an absolute requirement. We use a bottom-up approach and model energy demand for devices at oil and gas platforms with hourly time resolution. In this way, we can utilise the potential shifting of loads, such as for water injection, to balance volatile renewable energy better.

We conduct sensitivity analysis on $\mathrm{CO}_{2}$ tax, $\mathrm{CO}_{2}$ budget and the capacity of PFS. Based on the results, we address the value of OEHs in terms of system costs, energy losses and emissions.

The contributions of the paper are: (1) an integrated investment and operational model is developed with the following features: (a) OEHs are modelled for a large-scale offshore energy system and (b) the hourly devicelevel energy consumption of platforms is modelled, and (2) we analyse the value of OEHs in the North Sea offshore energy system transition towards zero-emission energy supply.

The outline of the paper is as follows: Section 2 introduces the background regarding offshore energy system decarbonisation and offshore oil and gas production. Section 3 gives the problem description followed by modelling strategies and assumption. Section 4 presents the model for the case study. Section 5 states the results and analysis of the case study. Section 6 concludes the paper and suggests further research.

\section{Background}

This paper tries to find the optimal system design of the NCS energy system for decarbonisation for a future point in time. We consider several decarbonisation solutions, including offshore wind, offshore solar, OEHs and PFS. As our focus is on the NCS, special attention is made to oil and gas recovery. In the following, we present background knowledge on offshore energy system decarbonisation approaches and the production process of offshore platforms.

\subsection{Offshore energy system decarbonisation}

$\mathrm{CO}_{2}$ tax is an important instrument for stimulating offshore energy sys-

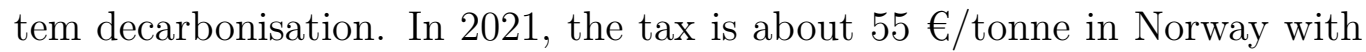
an ambition to increase it to $200 €$ /tonne by 2030. In addition, the EU Emissions Trading System is a "cap and trade" system that also includes the emissions on the NCS [36]. Carbon tax and the emissions trading system make a total carbon price of approximately $100 € /$ tonne. In this context, oil 
and gas companies are undertaking considerable investments in decarbonisation solutions to address climate goals, such as PFS and offshore wind. Oil and gas companies on the NCS have set climate targets. For example, Equinor and Vår Energy aim to reduce greenhouse gas emission by $40 \%$ by 2030 and near zero emission by $2050[8,55]$.

There are four general approaches to reduce offshore $\mathrm{CO}_{2}$ emissions. The first approach is to reduce $\mathrm{CO}_{2}$ emissions by improving reservoir drainage and processing energy efficiency. Water injection and gas injection are common reservoir drainage strategies used on the NCS. Pumping, compression and separation are major processes for handling produced fluids in a processing system. Injection and processing account for more than half of the power consumption at the fields on the NCS.

Secondly, it is possible to increase the energy efficiency of gas turbines. Due to security of supply requirements, gas turbines usually operate with a margin, which leads to a low efficiency of around 33\% [23]. Adding bottoming cycles to the existing gas turbines can improve their energy efficiency. However, unlike an onshore energy system, weight and space limitation of an offshore installation restrict extra devices like a bottoming cycle.

The third approach is to supply zero emission or low emission energy to offshore oil and gas platforms. This includes PFS, switching fuel from natural gas to ammonia or hydrogen and connecting offshore wind farms to platforms. In the past years, several offshore fields have received PFS via HVDC/HVAC cables [42, 37].

The cost of abating $\mathrm{CO}_{2}$ emissions by taking PFS can vary from less than 100 to almost $800 €$ /tonne [38]. Many abatement projects bringing PFS, are in their planning phase highly unprofitable even considering Norway's plan to increase $\mathrm{CO}_{2}$ tax to $200 €$ /tonne in 2030. Besides, due to the capacity limits of the onshore system, the available power is limited in some cases.

Offshore wind is another technology to supply clean power to platforms. Equinor's Hywind Tampen project aims to be operational by 2022 [7]. The combination of an offshore platform with a wind farm represents a potential good match for the offshore petroleum sector's desire for renewable energy with the offshore wind power industry's desire for an early market [47]. The stability and control issues for an isolated offshore energy system consisting of a wind farm and five platforms were addressed in [47]. Integrating large wind turbines into a stand-alone platform is theoretically possible but requires more operational and economic work to prove its feasibility [19]. In [25], a simulation model that connects a petroleum installation cluster in the 
Norwegian part of the North Sea to the Norwegian power grid via HVDC cables was presented. They found that local wind power production matching the offshore power demand improves both voltage- and frequency-stability in offshore system.

A framework for determining optimal offshore grid structures for wind power integration and power exchange named Net-Op was presented in [51]. The authors used a MILP model of the power grid to find optimal grid expansions for connecting offshore wind farms and increasing the transnational power exchange capacity in Europe. They pointed out that a capacity expansion model needs to include a power market model to capture the improved operation of the power system because of the transmission capacity expansion. An extension of Net-Op that takes into account investment cost, variability of wind/demand/power prices, and the benefit of power trade between countries/price areas was presented in [46].

The fourth approach is to deploy carbon capture and storage. Storing $\mathrm{CO}_{2}$ in stable underground formations, e.g., old and stable oil reservoirs, has a relatively long history. Since 1996, nearly one million tonnes of $\mathrm{CO}_{2}$ per year have been separated during the natural gas process from the Sleipner Vest field and stored in the Utsira formation [37].

The first two approaches have a limited impact on emission reduction. Whereas the third and fourth approaches can give up to $100 \%$ reduction.

The use of OEHs may allow for better harnessing offshore wind to supply more stable energy to platforms in the short run and export clean energy to the continent in the long run. Using energy hubs for coping with wind power volatility was discussed in [56]. Energy hubs can become sustainable pillars by improving cost-competitiveness of wind power [50]. In addition, the energy hub concept was also used to increase the energy flexibility in buildings [41] and electricity markets [40]. Techno-economic analysis of offshore energy islands was presented in [53]. A separate offshore bidding zone can lead to a more efficient offshore energy system with OEHs [21]. An offshore artificial power-to-gas island can produce and transport hydrogen through natural gas pipelines [14]. Energy-hub-based electricity system design for an offshore platform considering $\mathrm{CO}_{2}$ mitigation was presented in [57]. We refer the readers to $[30,31]$ for comprehensive reviews of the research works on energy hubs. There is a lack of research on the environmental value of OEHs. Besides research, real-world $\mathrm{OEH}$ projects will be carried out [4, 34, 32]. 


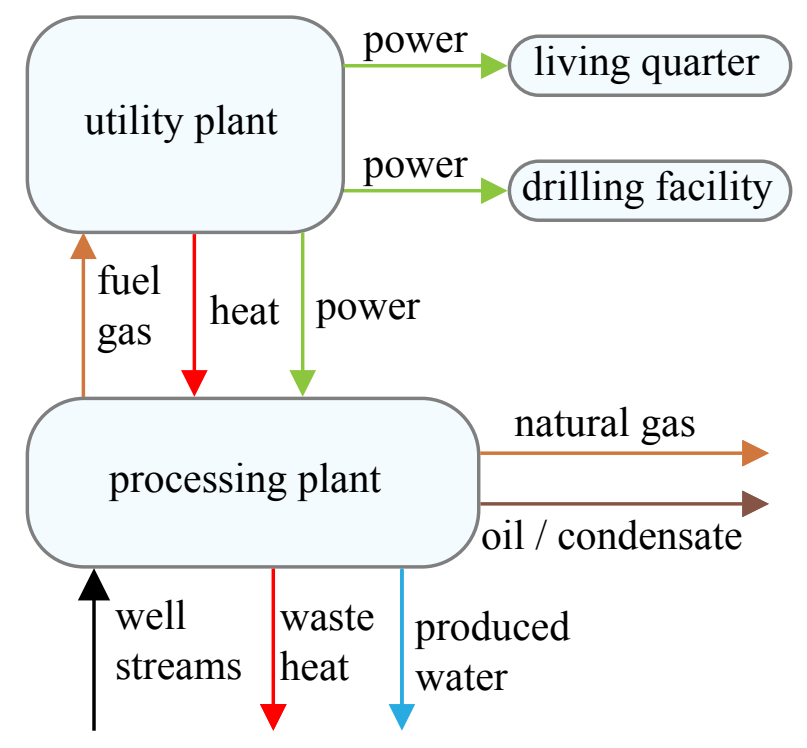

Figure 3: Schematic of a topside structure of a typical North Sea oil and gas platform, adapted from [54].

\subsection{Offshore oil and gas fields}

Offshore oil and gas field development and production involve multibilliondollar investments and profits [3]. A large number of studies have been carried out for offshore oil and gas fields production optimisation [16] and planning of offshore oil and gas infrastructures [17, 48]. From the studies, we can see that platforms and fields vary a lot due to, amongst others, geological characteristics, reserves, and remaining lifetimes. In the following, we present a typical composition and production process of NCS platforms.

A North Sea oil field normally consists of topside structures and subsea production systems. A topside structure typically consists of a processing plant, a utility plant, drilling facilities, and a living quarter [54], see Figure 3. The production plant receives and processes well streams. A visualisation of the production process is presented in Figure 4. Major energy consumption takes place in the production plants. The energy demand of production plants is conventionally fulfilled by gas turbines located in the utility plant. In 2014, gas turbines with waste heat recovery units covered approximately $90 \%$ of all heat demand for operations on the NCS [26]. 


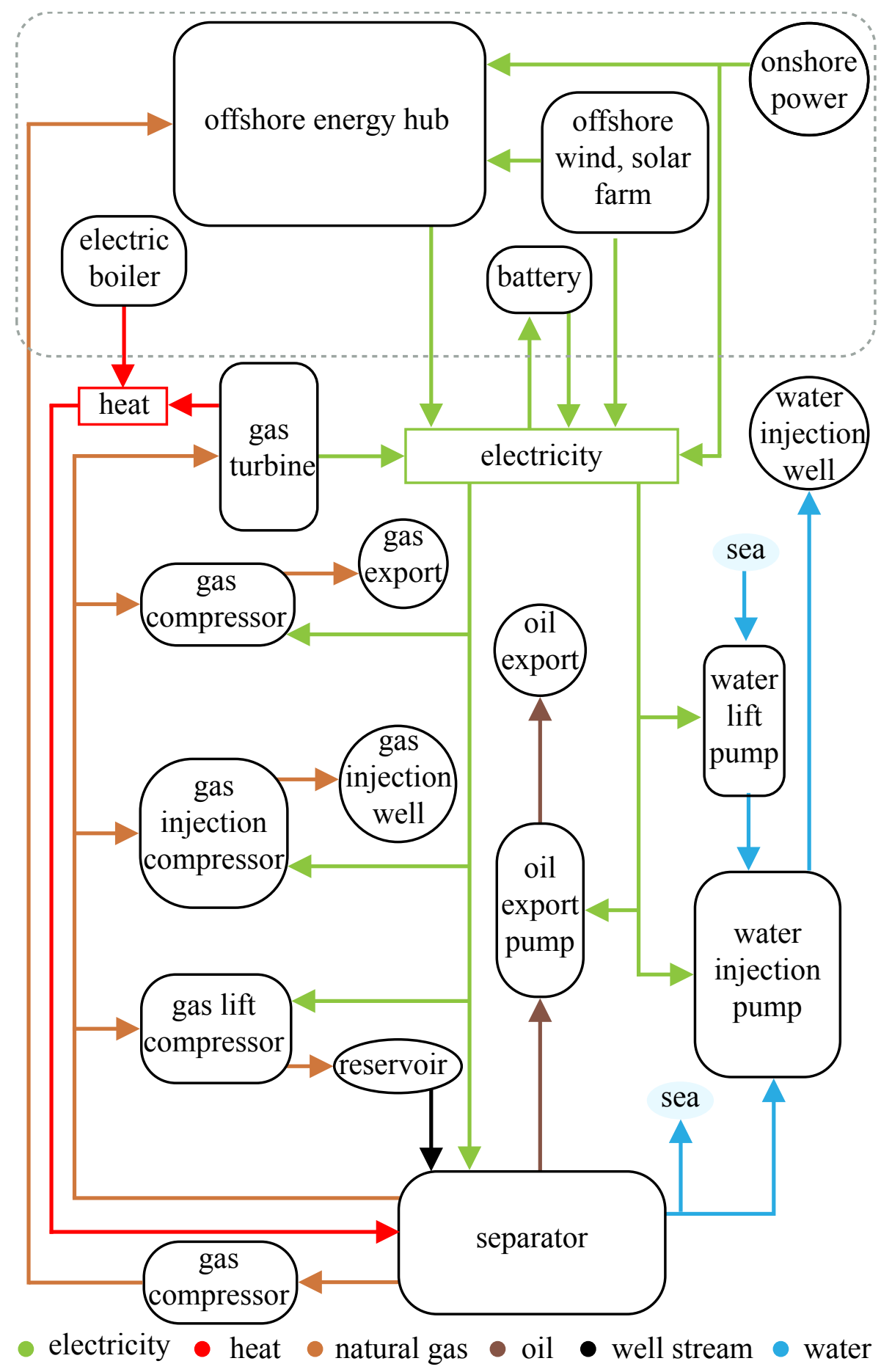


Figure 4: Schematic of a potential decarbonised offshore field production process. A three-stage separator train separates well streams into produced water, oil, condensate and gas. Typically the first stage separator takes out most of the water and gas at arrival conditions. Fuel gas is taken from the first stage separator. The residual mix of oil, gas and water is heated before entering the second stage separator. Produced water is purified and discharged, and in some cases, reinjected into water injection wells to maintain reservoir pressure. Water lift pumps will lift seawater for reinjection if needed. Produced oil is pressurised by pumps and exported. Produced gas is used as fuel gas, compressed and exported, reinjected via dedicated wells for enhanced oil recovery or injected into the same wells for gas lift.

The grey dotted box includes the potential processes for decarbonisation. See Figure 2 for a visualisation of the processes in an $\mathrm{OEH}$.

\section{Problem description}

First, this section introduces the proposed offshore energy system planning problem with OEHs. Then we present the time and geographical structures with the aim of reducing computational time of a potentially big problem. Finally, we give the modelling assumptions.

The problem under consideration aims to make optimal investment and operational decisions for the NCS energy system with OEHs, based on the energy demand captured by the operational model. By solving such a problem, we aim to find out under what conditions OEHs may benefit the system and how OEHs operate with the rest of the system.

To model hourly energy demand, we consider: (a) separators; (b) pumps: water injection pumps, water lift pumps, oil export pumps; (c) compressors: gas injection compressors and gas export compressors. These devices have existing capacities, and no investment will be made in them. Moreover, we assume that device efficiency, flow inlet/outlet pressures and hourly mass flow are given.

For the investments in decarbonisation solutions, we consider: (a) offshore renewable energies (offshore wind and offshore solar); (b) OEHs (electrolysers, hydrogen storage facilities and fuel cells); (c) subsea cables (HVAC, HVDC and offshore and onshore converter stations); (d) electric boilers; (e) platform located batteries. The capital expenditures, fixed operational costs are assumed to be known.

The problem is to determine (a) capacities of decarbonisation technologies and (b) operational strategies that include scheduling of generators, storage and approximate power flow among regions to meet the energy demand with 
minimum overall investment, operational and environmental costs.

\subsection{Modelling strategies and assumptions}

A multilevel control hierarchy was defined in [11], and they argued that the repetitive use of static models could solve many important petroleum production optimisation problems. We develop a multi-period MILP model for an integrated investment planning and operational problem that combines short-term and long-term control hierarchies. We use aggregation, clustering and time sampling [1] to address the multi-time-scale aspects [20] and solve a large-scale instance.

\subsubsection{Time structure of the problem}

The investment problem is optimised over a long-term horizon, e.g., a few decades. The operational problem is optimised on an hourly basis based on investment decisions. To combine these two control hierarchies without increasing much the computational time, we select $S$ representative slices, each containing $h$ hours, and scale them up to represent a whole operational year. A visualisation of the time structure is presented in Figure 5.

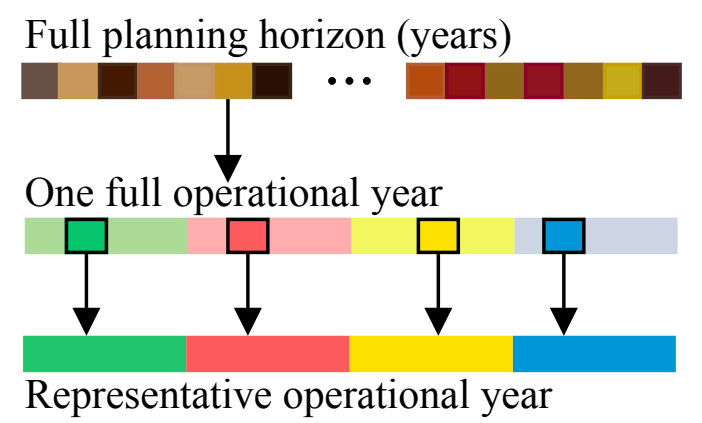

Figure 5: Illustration of combined hierarchies, (adapted from [52]).

\subsubsection{Geographical structure of the problem}

The problem potentially consists of many regions, and we implement a k-means cluster method based on the locations of fields to reduce the problem size. We have two considerations when deciding the number of clusters. Firstly, we assume the OEH connects the surrounding fields via HVAC cables; thus, only fields with a feasible transmission distance (up to $100 \mathrm{~km}$ ) are considered. Secondly, we assume that the cluster centres 
are the locations for OEHs. We prevent clusters with too few fields. For each cluster, we aggregate the individual fields into one bigger field with a distance to the OEH equal to the average distance of the individual fields and connect fields to OEH in hub-and-spoke form. Currently, we do not consider the interconnection among fields and clusters, resulting in reasonably simple network topology, exemplified in Figure 1.

\subsubsection{Assumptions}

We assume that each platform is a typical North Sea platform with production processes as presented in Figure 4. The energy consumption of pumps, compressors and separators can be formulated as a function of flow rate, pressure and temperature. For simplicity, we assume the pressure levels and temperatures to take values that are typical on the North Sea, leading to a linear formulation. Kirchhoff voltage law is omitted, and the model is an energy flow model. We assume no mass loss during production.

\section{Mathematical model}

This section presents a deterministic MILP formulation for the multicarrier energy system investment planning problem with high a degree of operational details. The complete nomenclature of the model can be found in Appendix A. The model includes a long-term investment planning horizon and a short-term operational horizon. The integrated investment planning and operational model is partially based upon the LP model developed in [27]. To improve the representation of the fixed capacity independent investment costs, we make use of integer variables. The planner is dedicated to the NCS energy system.

\subsection{Objective function}

$$
\min f(\mathbf{x})+\kappa \sum_{i \in \mathcal{I}} g\left(x_{i}, c_{i}\right)
$$

The objective function Equation (1) is to minimise the total investment $(f(\mathbf{x}))$ and operational $\left(\kappa \sum_{i \in \mathcal{I}} g\left(x_{i}, c_{i}\right)\right)$ costs over the planning horizon. The operational cost $g\left(x_{i}, c_{i}\right)$ is described in Section 4.3, where $x_{i}$ and $c_{i}$ are vectors containing capacities and costs information respectively. 


\subsection{Investment planning constraints}

$$
\begin{aligned}
& f(\mathbf{x})=\sum_{i \in \mathcal{I}_{0}}\left(\sum_{p \in \mathcal{P}} \sum_{z \in \mathcal{Z}}\left(C_{p i}^{P I n v} x_{p z i}^{P I n s t}+C_{p i}^{P F I n v} \gamma_{p z i}^{P}\right)\right. \\
& \left.+\sum_{l \in \mathcal{L}}\left(C_{l i}^{L I n v} X_{l}^{L g t h} x_{l i}^{L I n s t}+\left(C_{l i}^{L L I n v} X_{l}^{L g t h}+C_{l i}^{L F i n v}\right) \gamma_{l i}^{L}\right)\right) \\
& +\kappa \sum_{i \in \mathcal{I}}\left(\sum_{p \in \mathcal{P}} \sum_{z \in \mathcal{Z}} C_{p i}^{P F i x} x_{p z i}^{P A c c}+\sum_{l \in \mathcal{L}} C_{l i}^{L F i x} X_{l}^{L g t h} x_{l i}^{L A c c}\right) \\
& x_{p z i}^{P A c c}=X_{p z}^{P H i s t}+\sum_{i \in \mathcal{I}_{i}} x_{p z i}^{\text {PInst }}, \quad \quad p \in \mathcal{P}, z \in \mathcal{Z}, i \in \mathcal{I} \\
& x_{l i}^{L A c c}=X_{l}^{L H i s t}+\sum_{i \in \mathcal{I}_{i}} x_{l i}^{L I n s t}, \quad l \in \mathcal{L}, i \in \mathcal{I} \\
& 0 \leq x_{p z i}^{P I n s t} \leq \gamma_{p z i}^{P} M_{p}^{P}, \quad p \in \mathcal{P}, z \in \mathcal{Z}, i \in \mathcal{I}_{0} \\
& 0 \leq x_{l i}^{L I n s t} \leq \gamma_{l z i}^{L} M_{l}^{L}, \quad l \in \mathcal{L}, i \in \mathcal{I}_{0} \\
& 0 \leq x_{p z i}^{P A c c} \leq X_{p z}^{P \text { Max }}, \quad p \in \mathcal{P}, z \in \mathcal{Z}, i \in \mathcal{I} \\
& 0 \leq x_{l i}^{L A c c} \leq X_{l}^{L M a x}, \\
& l \in \mathcal{L}, i \in \mathcal{I} \\
& \sum_{i \in \mathcal{I}_{0}} \gamma_{p z i}^{P} \in\left\{0,1,2, \ldots, \bar{N}_{p z}\right\}, \quad p \in \mathcal{P}, z \in \mathcal{Z} \\
& \sum_{i \in \mathcal{I}_{0}} \gamma_{l i}^{L} \in\left\{0,1,2, \ldots, \bar{N}_{l}\right\}, \quad l \in \mathcal{L} \\
& x_{p z i}^{P I n s t}, x_{l i}^{L I n s t}, x_{p z i}^{P A c c}, x_{l i}^{L A c c} \in \mathbb{R}_{0}^{+} \text {, } \\
& \gamma_{p z i}^{P}, \gamma_{l i}^{L} \in \mathbb{Z}_{0}^{+} \text {. }
\end{aligned}
$$

Equation (2a) calculates the total capacity dependent investment costs, fixed operating and maintenance costs and fixed capacity independent investment costs. We define $\mathbf{x}$ to be a vector collecting available capacities of all technologies $(\mathcal{P})$ and branches $(\mathcal{L})$ for all operational years $(\mathcal{I})$. Constraints $(2 \mathrm{~b})$ and $(2 \mathrm{c})$ represent that the available capacity of a technology $\left(x_{p z i}^{P A c c}\right)$ or a branch $\left(x_{l i}^{L A c c}\right)$ at an operational year equals to its historical capacity $\left(X_{p z}^{\text {PHist }}\right.$ or $\left.X_{l}^{\text {LHist }}\right)$ and the sum of newly invested capacities $\left(x_{p z i}^{P I n s t}\right.$ or $\left.x_{l i}^{L I n s t}\right)$ in its ancestor investment periods $\left(\mathcal{I}_{i}\right)$. An integer variable $\gamma_{p z i}^{P}$ gives the number of units of technology $p \in \mathcal{P}$, in location $z \in \mathcal{Z}$, in investment period $i \in \mathcal{I}_{0}$. We define $\gamma_{l i}^{L}$ to be the number of cables of branch $l \in \mathcal{L}$. Parameters $M_{p}^{P}$ and $M_{l}^{L}$ state the maximum capacity of a technology 
unit and a cable, respectively. Parameters $\bar{N}_{p z i}$ and $\bar{N}_{l i}$ give the maximum number of units that can be installed of technologies and cables, respectively.

\subsection{Generic operational constraints}

We now present the generic operational constraints in one operational year $i$. Note that we omit index $i$ in the operational model for ease of notation. We model oil and gas recovery as this is the most likely use in the short to medium term. The operational constraints can be modified for other use, e.g., offshore fish farming, maritime, transport, and others. The complete description of operational constraints is presented in Appendix B.

$$
\begin{aligned}
& g(x, c)=\sum_{s \in \mathcal{S}^{T}} \sum_{t \in \mathcal{T}} \sum_{z \in \mathcal{Z}} W_{s}\left(\sum_{p \in \mathcal{P}} C_{p}^{P} y_{p z t}^{P}+C^{\text {Shed }} y_{z t}^{\text {LShed }}+\right. \\
& \left.\sum_{g \in \mathcal{G}} C^{\mathrm{CO}_{2}} \rho_{g}^{E} y_{g z t}^{G}\right) \\
& 0 \leq y_{p z t}^{P} \leq x_{p z}^{P A c c}, \\
& p \in \mathcal{P}, z \in \mathcal{Z}, t \in \mathcal{T} \\
& -x_{l}^{L A c c} \leq y_{l t}^{L} \leq x_{l}^{L A c c} \text {, } \\
& l \in \mathcal{L}, t \in \mathcal{T} \\
& \sum_{g \in \mathcal{G}} y_{g t}^{G}+\sum_{l \in \mathcal{L}} A_{z l} y_{l t}^{L}+\sum_{f \in \mathcal{F}} y_{f z t}^{F}+y_{z t}^{L S h e d}+\sum_{r \in \mathcal{R}} y_{r z t}^{R}= \\
& Y_{z t}^{D}+\sum_{s \in \mathcal{S}}\left(y_{s z t}^{S+}-y_{s z t}^{S-}\right)+\sum_{e \in \mathcal{E}} y_{e z t}^{E}+y_{z t}^{G S h e d}, \quad z \in \mathcal{Z}, t \in \mathcal{T} \\
& l_{s z(t+1)}^{S}-l_{s z t}^{S}=\eta_{s}^{S} y_{s z t}^{S+}-y_{s z t}^{S-}, \\
& s \in \mathcal{S}, z \in \mathcal{Z}, t \in \mathcal{T} \\
& \sum_{e \in \mathcal{E}} y_{e z t}^{E}+\sum_{s \in \mathcal{S}^{H y}} \eta^{E F} v_{s z t}^{S H y-}= \\
& \sum_{s \in \mathcal{S}^{H y}} \eta^{E S} v_{s z t}^{S H y+}+\sum_{f \in \mathcal{F}} \eta^{E F} y_{f z t}^{F}, \\
& z \in \mathcal{Z}, t \in \mathcal{T} \\
& \sum_{s \in S^{T}} \sum_{z \in \mathcal{Z}} \sum_{t \in \mathcal{T}} \sum_{g \in \mathcal{G}} W_{s} \rho_{g}^{E} y_{g z t}^{G} \leq E^{\mathrm{CO}_{2}}, \\
& y_{p z}^{P}, y_{g z t}^{G}, y_{f z t}^{F}, y_{z t}^{L S h e d}, y_{r z t}^{R}, y_{s z t}^{S+}, y_{s z t}^{S-}, y_{e z t}^{E} \in \mathbb{R}_{0}^{+} \text {, } \\
& y_{z t}^{\text {Gshed }}, L_{s z t}^{S}, v_{s z t}^{S H y-}, v_{s z t}^{S H y+}, x_{p z}^{P A c c}, x_{l}^{L A c c} \in \mathbb{R}_{0}^{+} \text {. }
\end{aligned}
$$

The operational cost function $g(x, c)$, which is included in the objective function Equation (1) for each operational year $i$, is described by Equation (3a) that includes total operating costs of all devices, energy load shedding costs and $\mathrm{CO}_{2}$ emissions costs. Vectors $x$ and $c$ contain capacities and costs 
information, respectively. Constraints (3b) and (3c) ensure devices $\left(y_{p z t}^{P}\right)$ and transmission lines $\left(y_{l t}^{L}\right)$ are within their capacities $\left(x_{p z}^{P A c c}, x_{l}^{L A c c}\right)$. Constraint (3d) gives the energy balance at each region, where $y_{g t}^{G}, y_{f z t}^{F}$ and $y_{r z t}^{R}$ are power generation of generators, fuel cells and renewables respectively. Moreover, we define $y_{e z t}^{E}$ to be the power that goes into electrolysers and $l_{s z t}^{S}, y_{s z t}^{S+}$ and $y_{s z t}^{S-}$ represent the storage level, input and output energy of storage facilities. The energy demand $Y_{z t}^{D}$ can be modelled corresponding to the specific sector, such as offshore platforms. The modelling of offshore platforms is described in details in Appendix B. Constraint (3e) states the storage balance of storage facilities. Constraint (3f) gives the hydrogen nodal balance of OEHs, where $v_{s z t}^{S H y-}$ and $v_{s z t}^{S H y+}$ are the hydrogen output and input of hydrogen storage facilities. Constraint (3g) restricts the total emissions. We only consider emissions from the generators, but the model can easily be extend to include other emissions. The complete MILP problem consists of Equations (1)-(3).

\section{Results}

We demonstrate the results of a static integrated investment planning and operational problem given by Equations (1)-(3) for a future point in time. The problem consists of 461, 108 continuous variables, 100 integer variables and 980,013 constraints. We implemented the model in Julia 1.6.1 using JuMP [6] and solved with Gurobi 9.1.2 [18]. We run the code on a MacBook Pro with $2.4 \mathrm{GHz}$ 8-core Intel Core i9 processor, with $64 \mathrm{~GB}$ of RAM, running on macOS 11.6 Big Sur. The Julia code and data for the case study have been made publicly available [58]. We solve the integrated investment and operational model given by Equations (1)-(3) to conduct sensitivity analysis on $\mathrm{CO}_{2}$ tax, $\mathrm{CO}_{2}$ budget and the capacity of PFS.

\subsection{Case study}

The case study is carried out on the North Sea part of the NCS, considering 66 fields. The problem consists of 77 regions, divided into 66 fields, 5 OHEs and 5 onshore buses. By using the clustering approach described in Section 3.1 we find that the system can be represented using 5 clusters and henceforth go from 77 regions to 15 regions. We assume that the power demand of platforms initially is entirely supplied by gas turbines, as only a limited number of platforms receives PFS. We construct four representative months with hourly resolution and scale them up to represent a whole year. 


\begin{tabular}{cccccc}
\hline & cluster1 & cluster2 & cluster3 & cluster4 & cluster5 \\
\hline Emission distribution & $7.2 \%$ & $5.5 \%$ & $44.7 \%$ & $11.2 \%$ & $31.3 \%$ \\
\hline
\end{tabular}

Table 1: Emission distribution by cluster.

The operational data of oil and gas industry is sensitive and usually not disclosed to the public. Aggregated data such as monthly or yearly production of petroleum on the NCS can be obtained from [37]. One can also find monthly production and injection data for each field from [35] and [39]. Neither of these can be directly used as inputs for this study due to the time resolution difference. Therefore, reasonable data generation is necessary. Raw data is collected from (a) Norne (1998 - 2006) and Volve (2008 - 2016) fields with hourly production and injection data from [49] and (b) monthly production and injection data of each field from [35]. We come up with a data generation method that considers the lifetimes of offshore fields [58].

We define a base case (Base) with offshore renewables, electric boiler, battery and PFS as investment options. We then use this case as a benchmark to check against the case with OEHs. The full model given by Equations (1)(3) takes approximately 2 hours.

\subsection{Energy system analysis}

In this section, we present results on energy consumption and $\mathrm{CO}_{2}$ emission of the initial system. By post-processing, we verify that the energy consumption of platforms is of the same order of magnitude as reported numbers. The resulted $\mathrm{CO}_{2}$ emission is $5.51 \mathrm{Mt} / \mathrm{yr}$. In comparison, the reported total emission of the relevant fields was 6.89 Mt in 2019 [39]. We expect emissions from the model to be lower than 6.89 Mt since not all emission sources are considered. Based on [33], one could assume that the major processes considered in this study make up about $80 \%$ of the total load. Therefore, 5.51 Mt yearly emission is within the correct range, implying that the energy load modelling is relatively accurate.

From Figure 6, we can see that the power output of the Open Cycle Gas Turbine (OCGT) matches power demand at every operational period. Heat recovery of OCGTs is assumed the only heat source. Figure 7 shows that heat recovery of OCGTs provides more than enough heat due to high electricity generation. We can also see that energy consumption can vary significantly. A breakdown of electricity load is shown in Figure 8, gas export compressors dominate the power consumption in clusters $3-5$. Water injection is 


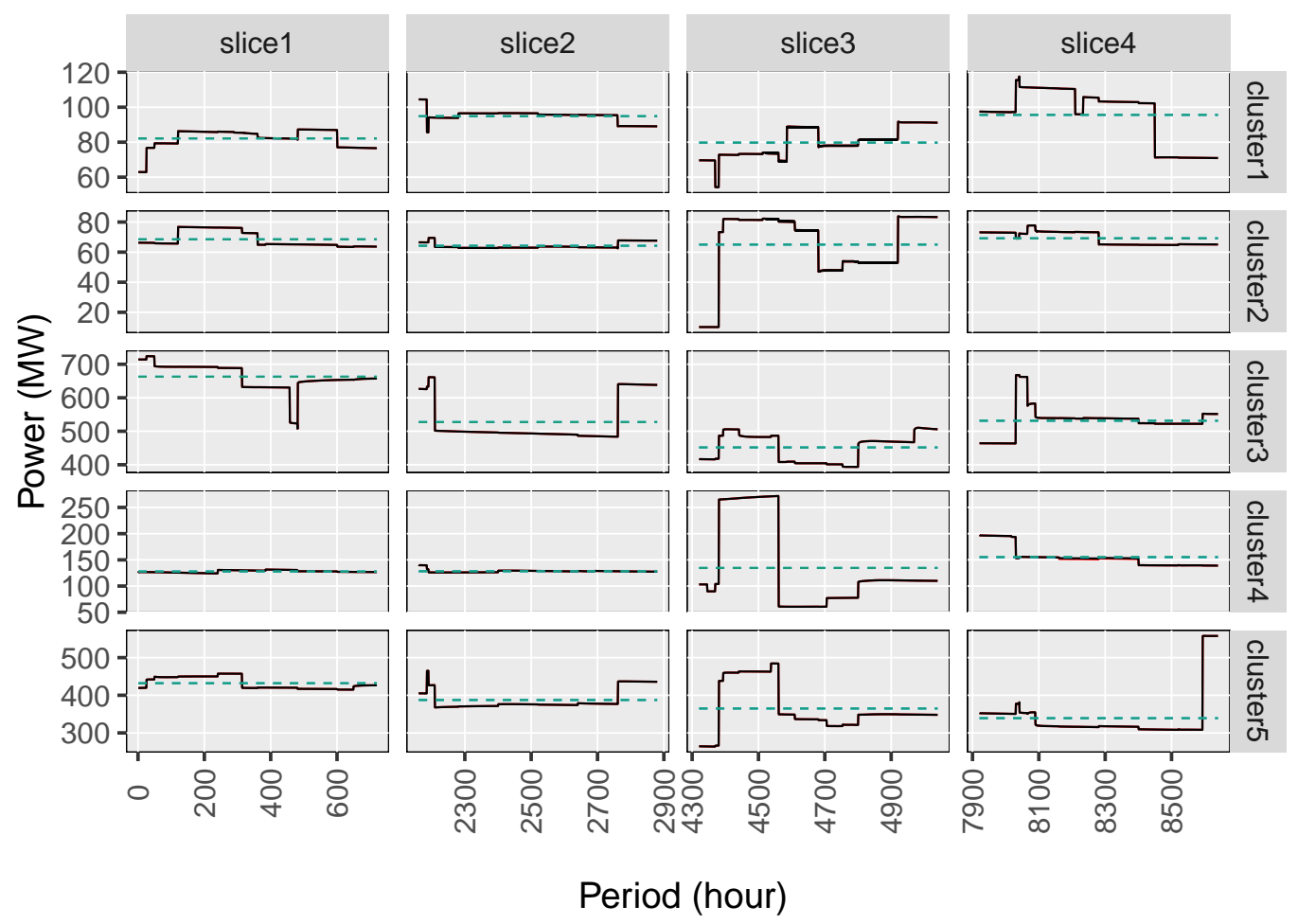

— ave OCGT power — ave power demand _ OCGT power — power demand

Figure 6: Power consumption and supply (Only two lines are observable since power supply and demand match exactly. OCGT power equals power demand at all times).

the largest power consumer in cluster 2 since there are some mature fields (e.g., Ekofisk) whose reservoir pressures are mainly maintained by water injection. OCGT is the only energy and emission source in the initial setup. Therefore, emission breakdown includes the emissions from the total energy consumption of each region. Cluster 1 has the second smallest share of the total energy consumption, with a considerable amount of power consumed by gas injection. The fields in cluster 1, such as Grane, have the third-highest gas injection level among the 66 fields. From Table 1, we find that emission mainly comes from the northern part of the North Sea. 


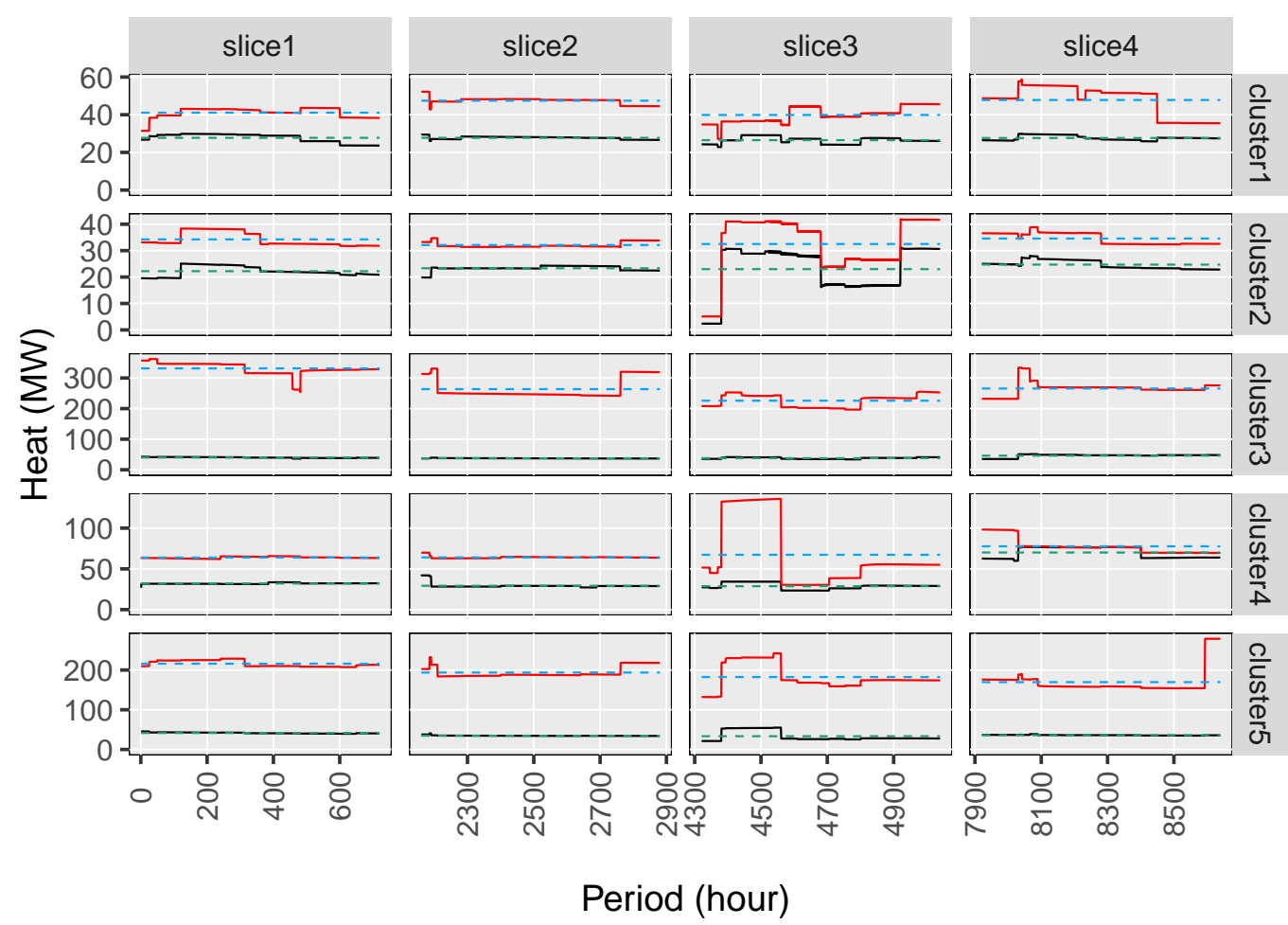

ave heat demand _ ave OCGT heat _ heat demand _ OCGT heat

Figure 7: Heat consumption and supply.

\subsection{Sensitivity analysis of $\mathrm{CO}_{2}$ tax}

This section presents the results of sensitivity analysis of $\mathrm{CO}_{2}$ tax. We introduce $\mathrm{CO}_{2}$ tax and still keep the carbon budget inactive. We increase the carbon tax from 55 to $500 €$ /tonne with a step size of $5 € /$ tonne. PFS capacity limits are estimated from $[38,44]$. Note that the cost of PFS may be underestimated since we only consider the costs of subsea cables, onshore and offshore converter stations and electricity bills. In reality, PFS projects may also face investment in onshore transmission lines or onshore power system capacity expansion. We analyse the results from two metrics, $\mathrm{CO}_{2}$ emission and energy loss. Energy losses are from conversions, transmission, and generation shed. The calculation is presented in Appendix C.

From Figure 9, we can see that $\mathrm{CO}_{2}$ tax as a single instrument may 

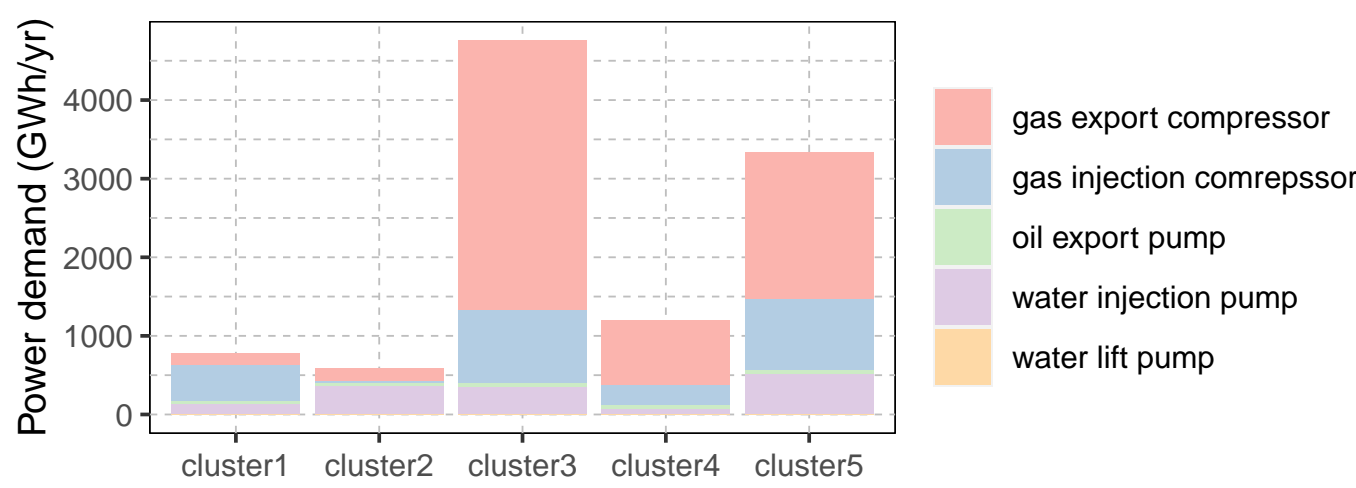

Figure 8: Power demand in a year.
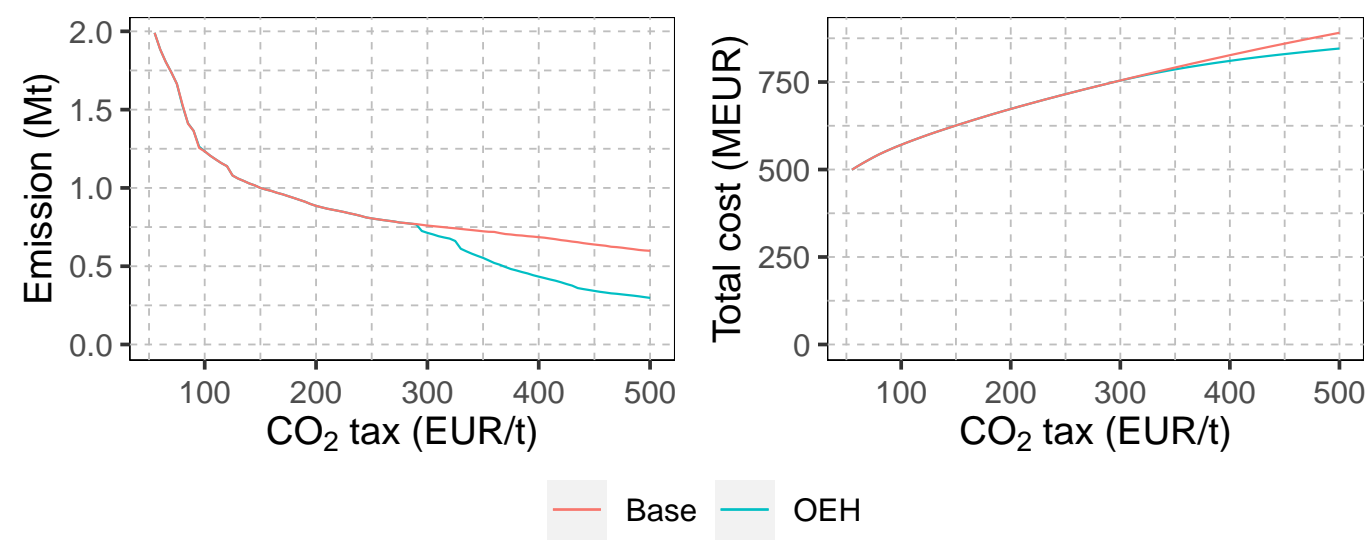

Figure 9: Emission and cost comparison $\left(\mathrm{CO}_{2}\right.$ tax sensitivity analysis).

not be enough to motivate a zero emission system. We also find that near zero emission can be achieved with a very high $\mathrm{CO}_{2}$ tax. Therefore, a hard carbon cap may be necessary for stimulating a zero emission system. When $\mathrm{CO}_{2}$ tax is $55 € /$ tonne, the system reduces about $64 \%$ of the initial emissions. Approximately $84 \%$ of the emissions can be cut if $\mathrm{CO}_{2}$ tax is increased to 200 $€ /$ tonne as planned. As OCGTs are replaced by renewable energy, energy loss is reduced as well. OEHs can potentially reduce up to around $50 \%$ more $\mathrm{CO}_{2}$ emission and $5 \%$ total cost than the case with only offshore wind and PFS (Base) at certain $\mathrm{CO}_{2}$ tax levels. From Figure 10, we find that energy loss during production counts for $11 \%$ of the energy loss. OCGTs lose $18 \mathrm{GWh}$ 

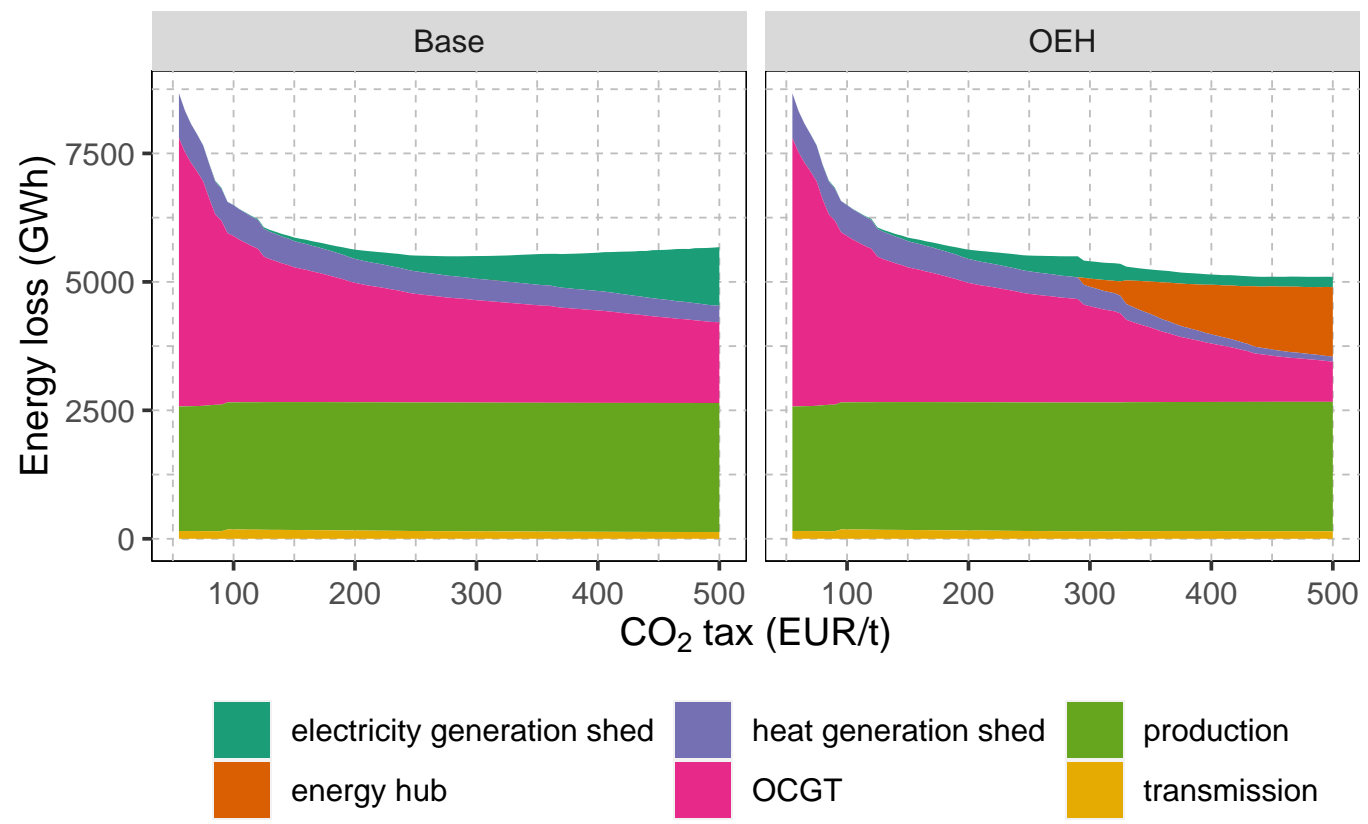

production

transmission

Figure 10: Energy loss $\left(\mathrm{CO}_{2}\right.$ tax sensitivity analysis).
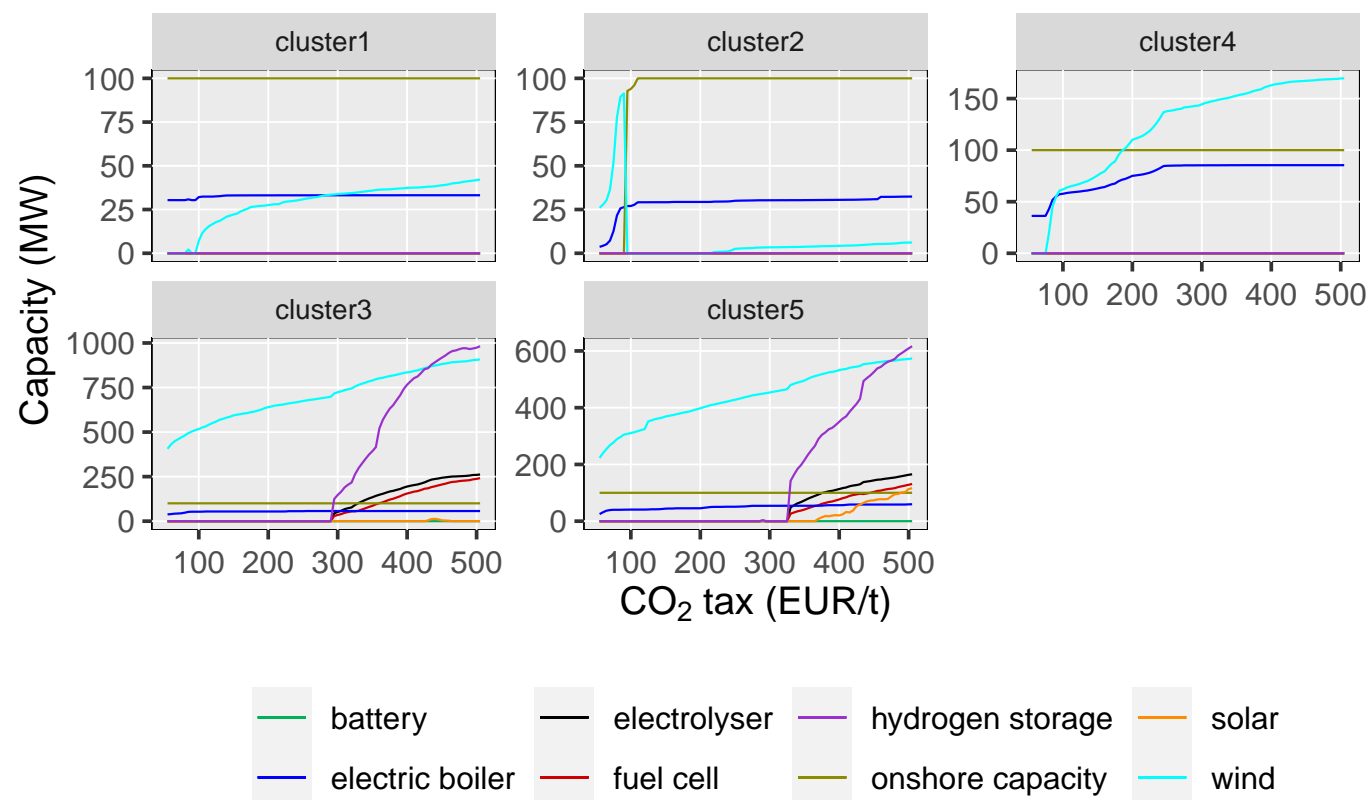

Figure 11: Capacities of technologies in each cluster $\left(\mathrm{CO}_{2}\right.$ tax sensitivity analysis), hydrogen storage is measured in tonne. 

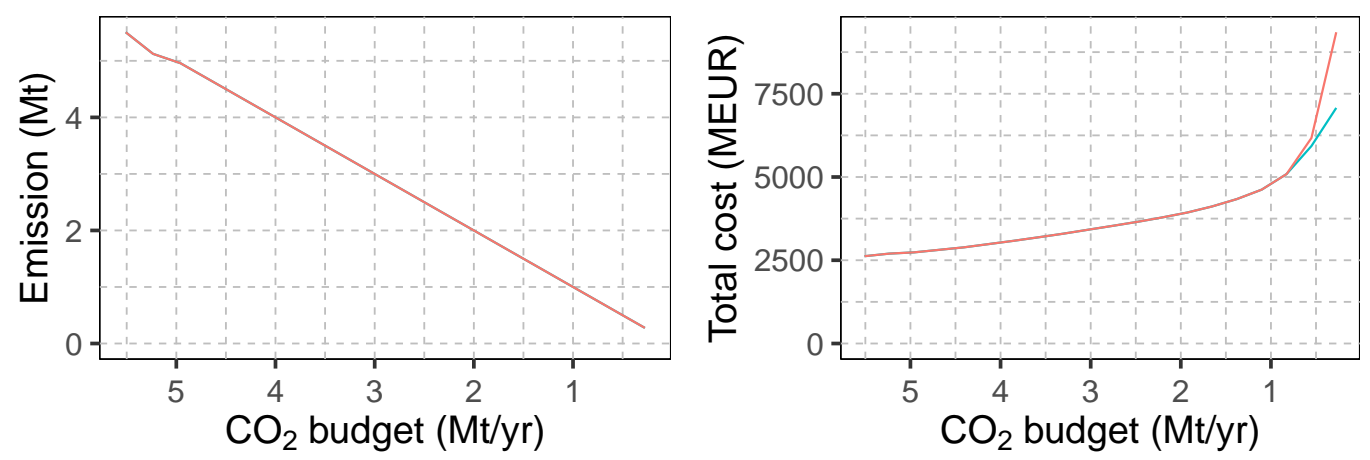

Base — OEH

Figure 12: Emission and cost comparison $\left(\mathrm{CO}_{2}\right.$ budget sensitivity analysis).

of energy during an operational year. As production from wind turbines replaces gas turbines, energy loss from OCGT is reduced. However, due to the lack of energy storage, electricity generation shedding increases because wind power is shed. We find that OEHs can effectively reduce electricity generation shedding although, it loses energy during conversion. Overall, energy loss is up to $10 \%$ lower in the case of OEHs compared with Base at certain tax levels.

From Figure 11, we find that different clusters show different levels of sensitivity to $\mathrm{CO}_{2}$ tax. Offshore wind is the first renewable energy solution that is added to the system. Electric boilers are soon needed as offshore wind replaces gas turbines partially. OEHs are installed when $\mathrm{CO}_{2}$ tax is above $290 €$ /tonne. Offshore solar is only added in clusters 3 and 5 under very high $\mathrm{CO}_{2}$ tax levels. OCGTs still operate even $\mathrm{CO}_{2}$ tax increases to 500 $\epsilon_{1}$ tonne. We can see that in a static planning problem, if $\mathrm{CO}_{2}$ tax is the only instrument and increases to $200 €$ /tonne as the government's plan in 2030, OEHs may not be necessary. However, $\mathrm{CO}_{2}$ tax combined with the EU emissions trading system may likely increase the total $\mathrm{CO}_{2}$ price to around $250-300 €$ /tonne, which is about the breakeven price of OEHs. In addition, the potential benefits of the OEHs may realise once they provide services to more sectors, such as exporting hydrogen for industries or transportation.

\subsection{Sensitivity analysis of $\mathrm{CO}_{2}$ budget}

For the $\mathrm{CO}_{2}$ budget, we use initial emission as the starting point and reduce it by $5 \%$ until it hits 0 . From Figure 12, we find that carbon cap is 

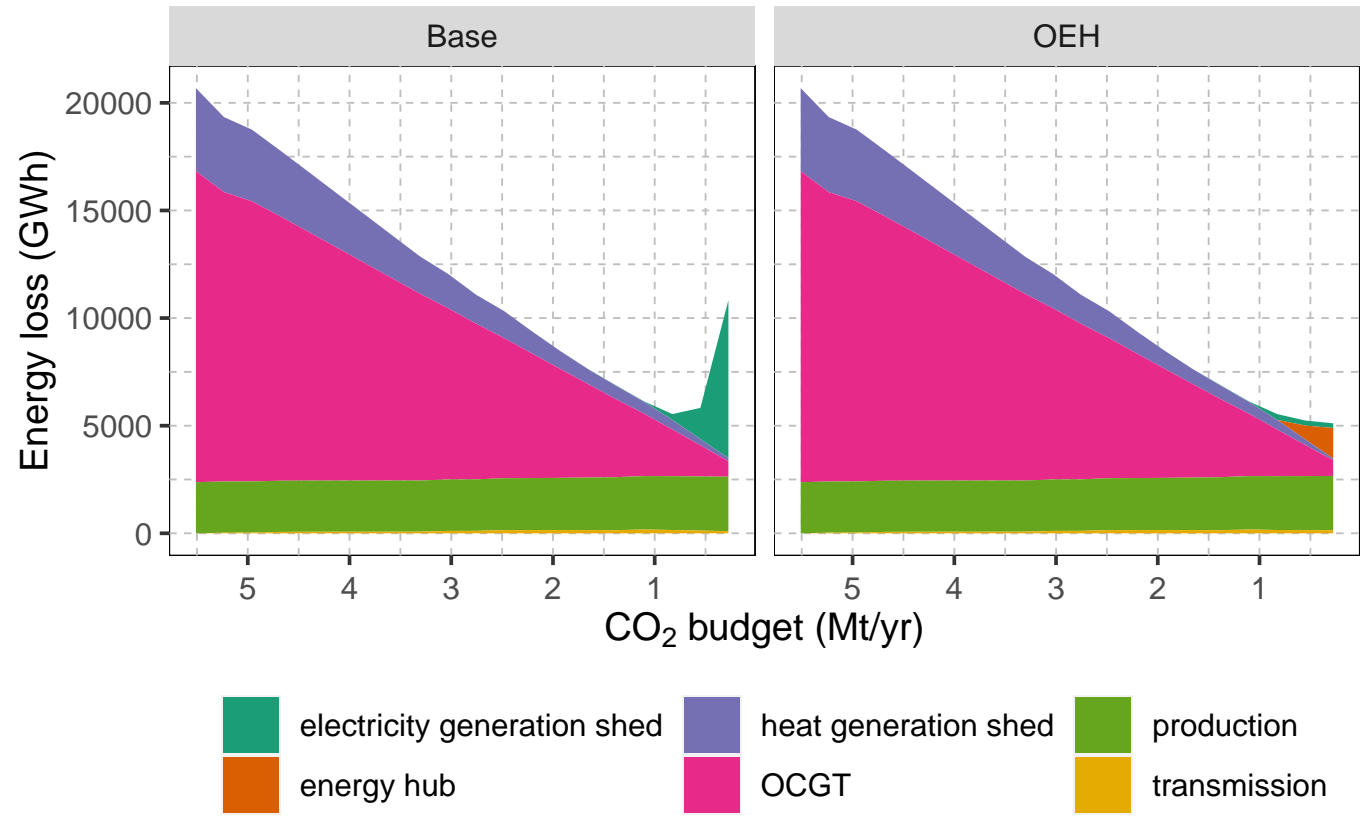

production

transmission

Figure 13: Energy loss $\left(\mathrm{CO}_{2}\right.$ budget sensitivity analysis $)$
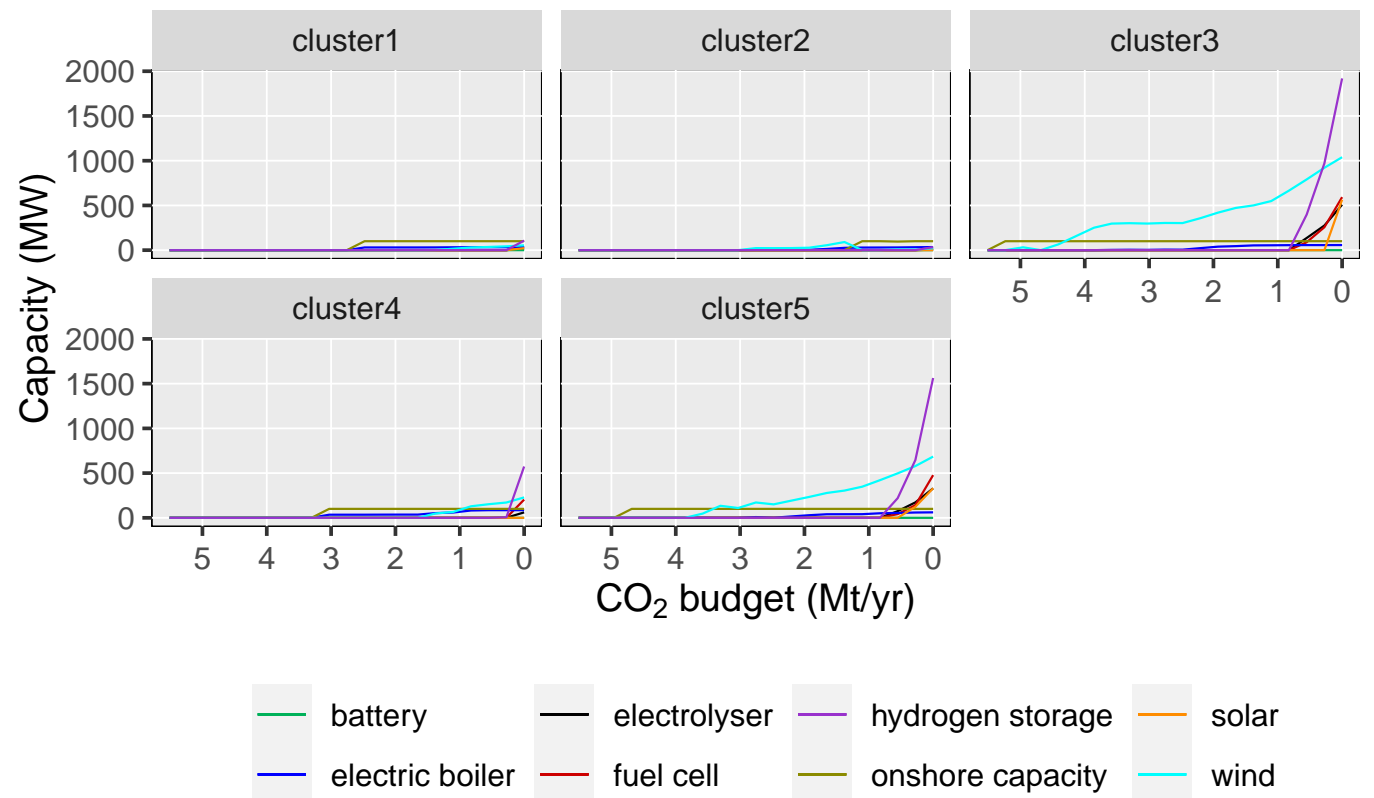

Figure 14: Capacities of technologies in each cluster $\left(\mathrm{CO}_{2}\right.$ budget sensitivity analysis), hydrogen storage is measured in tonne. 
binding most of the time and we rarely see that emissions reduce more than the carbon cap. Thus, there is no difference in actual emission in Base and the system with OEHs. However, the cost is $25 \%$ lower in a zero emission system with OEHs compared with Base.

We find that in a zero emission system without OEHs, energy loss is around 477 TWh due to $81 \mathrm{GW}$ of wind power capacity without storage. This may not be likely from happening since some forms of storage would be added to compensate for offshore wind in reality. From Figure 13, we can see a large amount of energy loss when reaching near zero emission system in Base. The energy loss in Base is 10,832 GWh in a near zero emission system that is about twice higher than that with OEHs. A large amount of wind power is installed to meet power demand at any time. Therefore, the same capacity of wind that can cope with peak demand hours will also generate surplus power during normal hours. This leads to increased energy losses as more wind replaces OCGT without proper energy storage. In the case of OEHs, wind power can be stored when excess power is generated. It is also worth noticing that in the energy system without an $\mathrm{OEH}$, energy storage is the battery on the platforms, which can be infeasible due to space and weight limitations. We observe that investments in batteries are only needed when approaching zero emission in Base. No battery is needed in a system with OEHs. In addition, the energy loss of OEHs is $27 \%$ of the total loss, and the loss during production is about $50 \%$ of the total.

From Figure 14, we find that cluster 3 receives PFS after a 10\% reduction of the carbon cap. Cluster 3 has the highest emission level but the shortest distance from shore. Therefore, taking PFS and partially electrify the fields in cluster 3 can help the system reduce $10 \%$ emission in a cost effective way. The system does not cut emissions proportionally in each cluster but cut emissions from clusters with the highest emission, such as cluster 3 and cluster 5. Therefore, it may be necessary to consider the whole NCS when conducting system planning rather than consider each cluster separately and reach sub-optimality. Cluster 2 is the most remote, more than $300 \mathrm{~km}$ from the shore; PFS is less economical than offshore wind. Therefore, offshore wind is added to cluster 2 when the carbon cap drops to $2.75 \mathrm{Mt} / \mathrm{yr}$. When the $\mathrm{CO}_{2}$ budget reduces to below $0.83 \mathrm{Mt} / \mathrm{yr}, \mathrm{CO}_{2}$ emissions are nearly zero in clusters 1 and 2 . However, the carbon cap needs to reduce to zero to shut down OCGTs completely in all clusters. Nearly 4,200 tonnes of hydrogen storage capacity is needed in a zero emission NCS energy system, and nearly half is installed in cluster 3. 

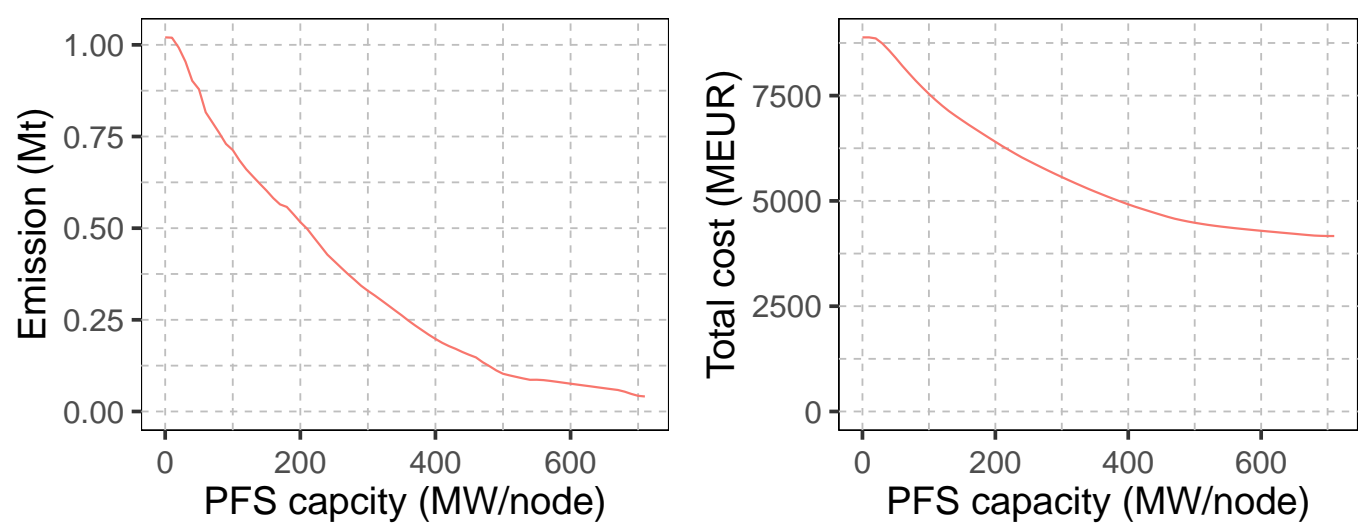

Figure 15: Emission and cost (PFS capacity sensitivity analysis, S1).

\subsection{Sensitivity analysis of the capacity of PFS}

We now present the results of sensitivity analysis of the capacity of PFS. The capacity of PFS affects the investments in offshore technologies. An onshore system has a limited capacity to transmit power offshore. Furthermore, onshore system expansion can vary the limitation. However, we do not consider onshore system expansion in this paper. Therefore, we conduct sensitivity analysis to reveal the relationship between onshore power system capacity and offshore decarbonisation technologies.

\subsubsection{Scenario 1 (S1)}

The first scenario is to fix the $\mathrm{CO}_{2}$ tax to $300 \epsilon_{\text {/ }}$ tonne and increase the PFS capacity of each onshore node from 0 MW to $1000 \mathrm{MW}$ with a 10 MW step. We find that the investment decisions remain the same if the PFS capacity is higher than 710 MW. Therefore, we only present the results from $0 \mathrm{MW}$ to $710 \mathrm{MW}$. From Figure 15, we can see that by having $710 \mathrm{MW}$ capacity in each onshore node, the system can achieve $0.05 \mathrm{Mt} / \mathrm{yr}$ emission and reduce nearly $50 \%$ of the total cost. However, increasing the capacity further does not cut emissions or costs further. Figure 17 shows that energy loss during transmission makes up 16\% of the total energy loss as we increase the onshore capacity. Electricity generation shed decreases as onshore capacity increases because PFS gradually replaces offshore wind, and less energy is lost from wind turbines. From Figure 16, we find that for onshore nodes that connect to cluster 1 and cluster 2, the needed onshore capacities are about $130 \mathrm{MW}$ and $110 \mathrm{MW}$, respectively. There are also 


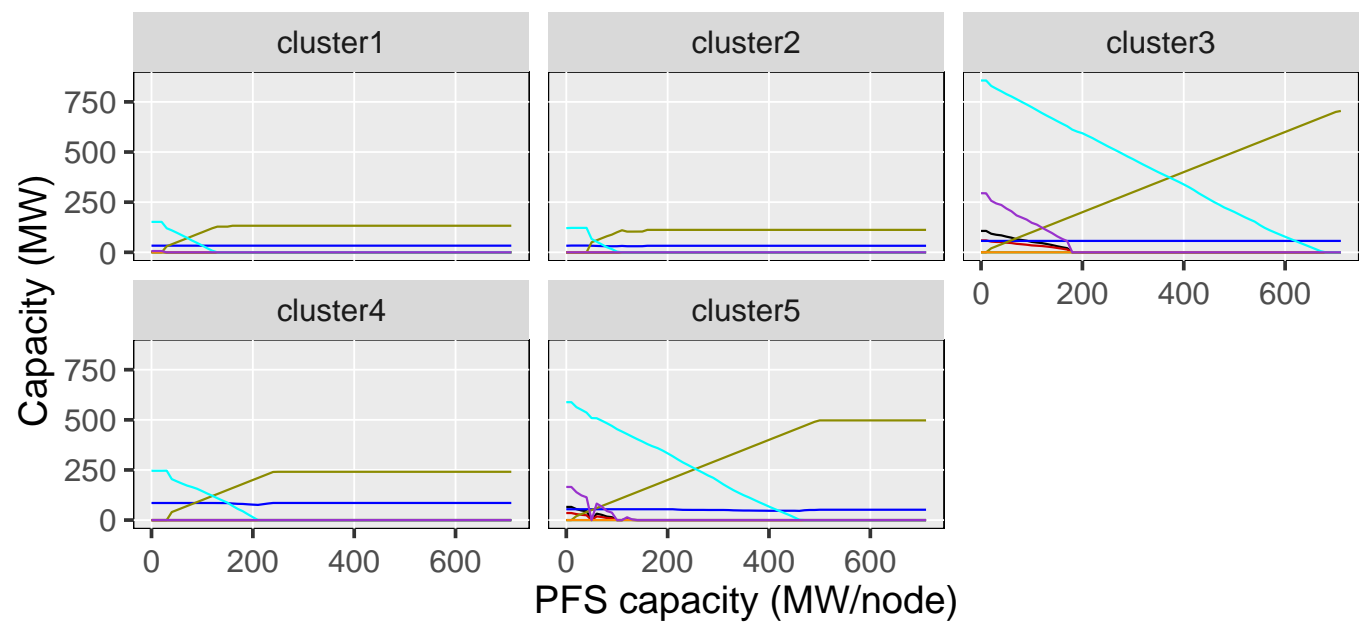

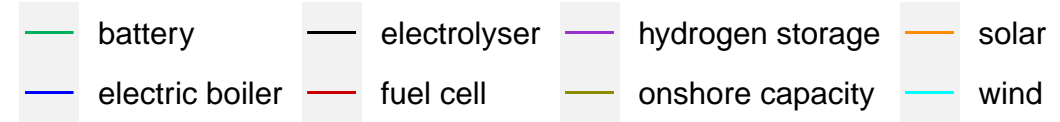

Figure 16: Capacities of technologies in each cluster (PFS capacity sensitivity analysis, $\mathrm{S} 1$ ), hydrogen storage is measured in tonne.

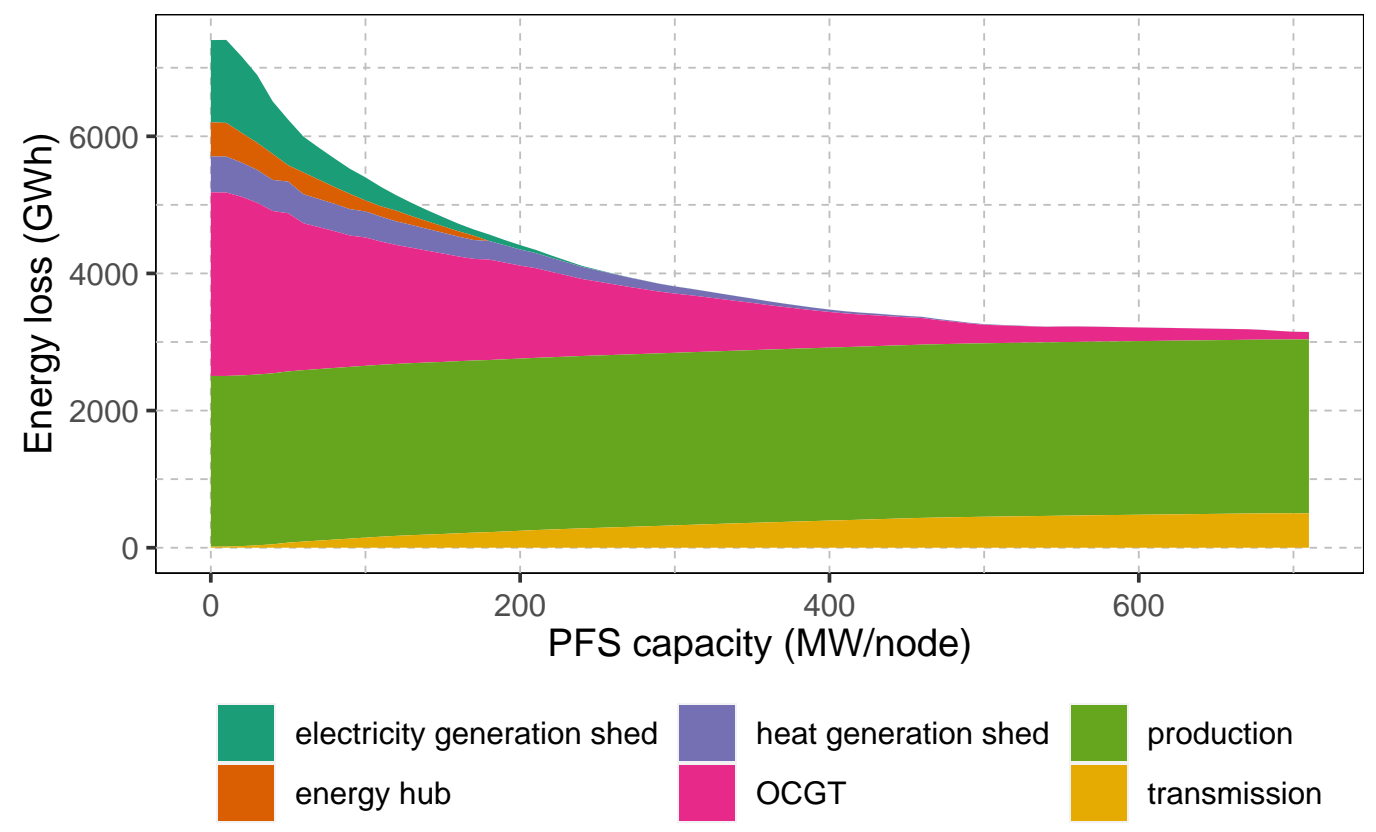

Figure 17: Energy loss (PFS capacity sensitivity analysis, S1). 

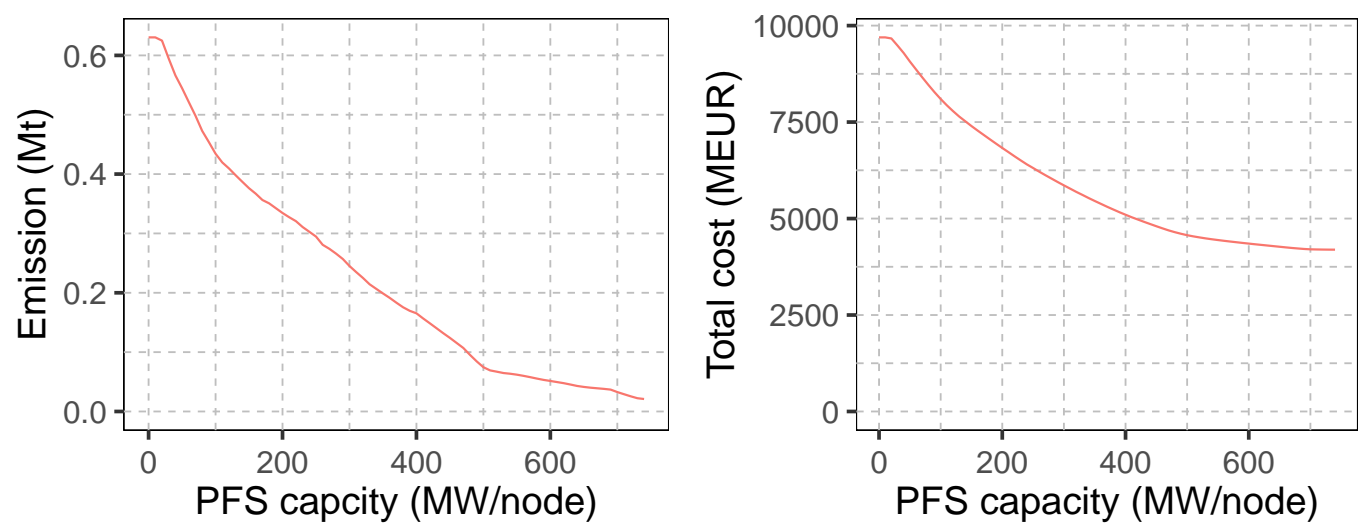

Figure 18: Emission and cost (PFS capacity sensitivity analysis, S2).

upper limits on the installed capacity of PFS in the other clusters. We also notice that OEHs are still needed in clusters 1, 3, 5 as we increase the onshore capacity. However, eventually, OEHs are not needed since PFS can provide more stable power and $\mathrm{OEH}$ with storage becomes less important.

\subsubsection{Scenario 2 (S2)}

In the second scenario, we fix the $\mathrm{CO}_{2}$ tax to $400 €$ /tonne. We increase the onshore capacity from 0 MW to $1000 \mathrm{MW}$ and present the results until 740 MW. From 18, we can see that without PFS, the system can achieve 0.63 $\mathrm{Mt} / \mathrm{yr}$ emissions under S2 condition. Increasing the onshore capacity brings down $43 \%$ of the cost and also cut emission further to near zero. Figure 20 shows that about $20 \%$ of the energy loss is from OEHs initially. OEHs are not needed when the onshore capacity increase to around $400 \mathrm{MW}$ for each node. By adding the installed PFS capacity shown in Figure 19, we find that a total onshore capacity of 1.75 GW may help the offshore energy system achieve near zero emission. We notice that the onshore system needs to provide an averagely of $1.4 \mathrm{GW}$ By checking the average power transmission of PFS, which might not be feasible without onshore system expansion.

\section{Conclusions and future work}

This paper presents a multi-carrier offshore energy system investment planning optimisation model with a high degree of operational detail to find cost-optimal solutions for decarbonising NCS energy supply. The major novelties and contributions are (1) we formulate OEHs in a MILP model for 

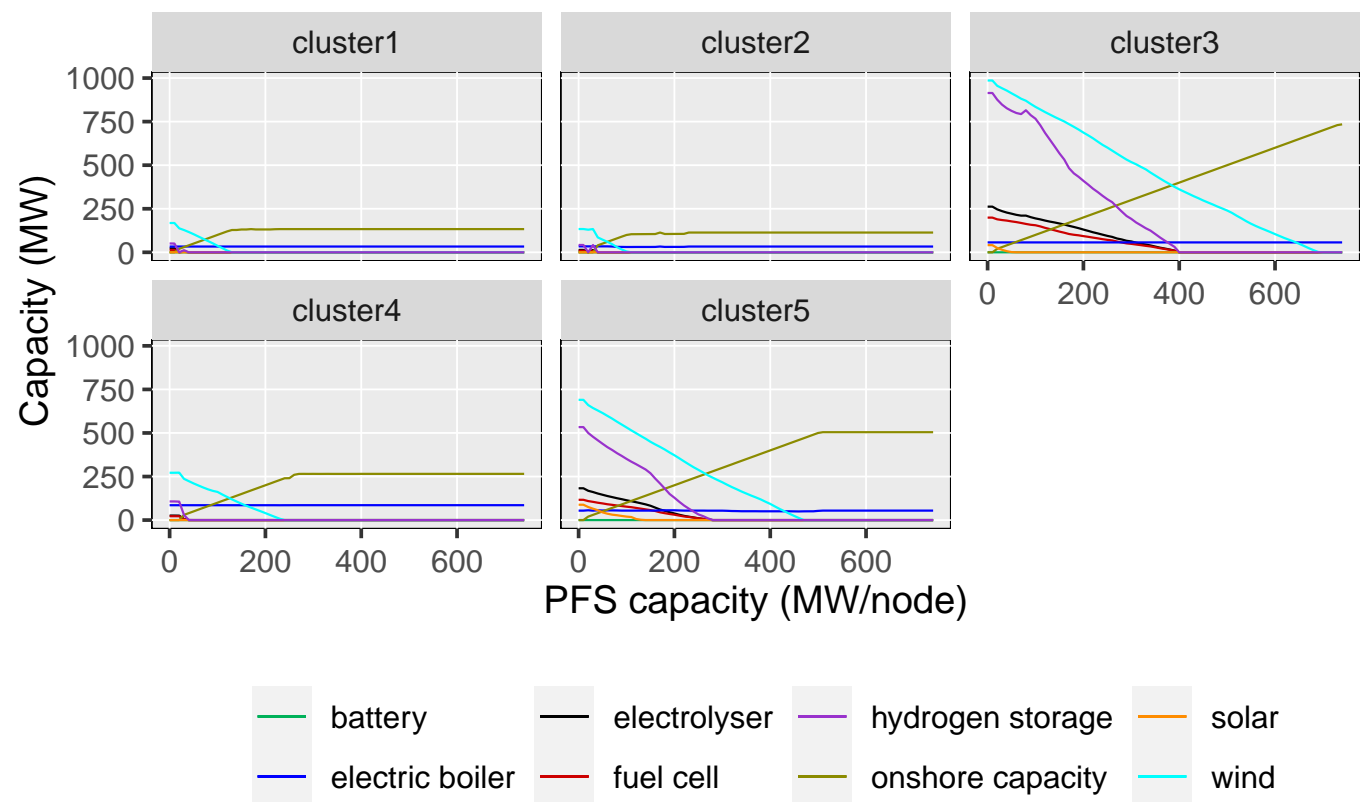

Figure 19: Capacities of technologies in each cluster (PFS capacity sensitivity analysis, $\mathrm{S} 2$ ), hydrogen storage is measured in tonne.

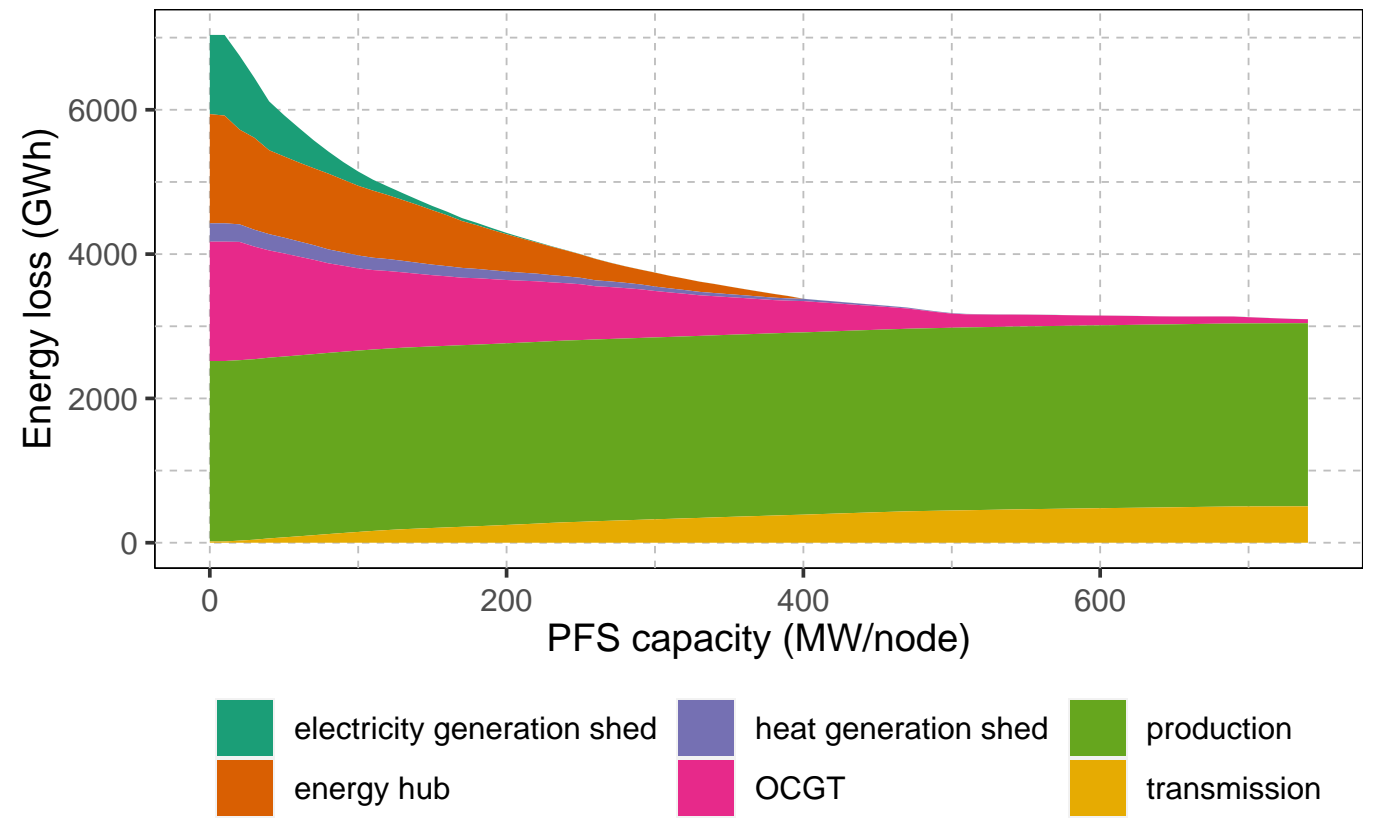

Figure 20: Energy loss (PFS capacity sensitivity analysis, S2). 
large-scale offshore energy system planning; (2) we model the device-level energy consumption of the offshore platforms with hourly time resolution on a large scale; and (3) We use an integrated MILP investment and operational model to conduct a large-scale analysis of the value of OEHs in the North Sea offshore energy system transition towards decarbonised energy supply. Results from our case study indicate that (1) OEHs can reduce up to $10 \%$ of the energy loss and $50 \%$ of the emissions with $\mathrm{CO}_{2}$ tax above $290 €$ /tonne; (2) OEHs can reduce energy loss by $65 \%$ in a near zero emission system; (3) a carbon budget may be necessary to enable a zero emission energy system in addition to $\mathrm{CO}_{2}$ tax; (4) the system cuts about $63 \%$ of the initial emissions when $\mathrm{CO}_{2}$ tax is $55 \epsilon_{1}$ /tonne, and approximately $84 \%$ of the $\mathrm{CO}_{2}$ emissions can be cut if $\mathrm{CO}_{2}$ tax is increased to Norway's target of $200 € /$ tonne; and (5) it may be necessary to consider the whole NCS when doing system planning towards zero emission rather than consider each region separately and reach sub-optimality.

Although the deterministic MILP model in this paper has led to many insights, there are possibilities for extensions. A deterministic optimisation model is not capable of representing load and supply uncertainties. Therefore, we aim to develop a stochastic optimisation model [2] and incorporate long-term and short-term uncertainties in future work. In addition, multiple investment stages are needed to represent the investment planning problem more realistically. Besides, we only consider using OEHs for fields decarbonisation, which makes OEHs seem less attractive than other technologies due to their high costs. However, OEHs can have various utilities such as energy provision to offshore fish farming, maritime and transport, and using the infrastructure past the lifetime of the oil and gas fields for purposes such as exporting hydrogen. These may motivate the investments in OEHs, and we aim to include some of the aspects in future. Finally, more work can be done on offshore network topology and the representation of the onshore power system.

\section{CRediT author statement}

Hongyu Zhang: Conceptualisation, Methodology, Software, Validation, Formal analysis, Investigation, Visualisation, Data curation, Writing - original draft, Writing - review \& editing. Asgeir Tomasgard: Conceptualisation, Supervision, Writing - review \& editing, Funding acquisition. Brage Rugstad Knudsen: Conceptualisation, Supervision, Writing - review \& 
editing. Harald G. Svendsen: Conceptualisation, Writing - review \& editing. Steffen J. Bakker: Conceptualisation, Writing - review \& editing. Ignacio E. Grossmann: Conceptualisation, Supervision, Writing - review \& editing.

\section{Declaration of competing interest}

The authors declare that they have no known competing financial interests or personal relationships that could have appeared to influence the work reported in this paper.

\section{Acknowledgements}

This work was supported by the Research Council of Norway through PETROSENTER LowEmission (project code 296207).

\section{Appendix A.}

\section{Nomenclature}

\section{Investment planning related sets}

$\mathcal{I}$ set of operational years

$\mathcal{I}_{0} \quad$ set of investment periods

$\mathcal{I}_{i} \quad$ set of investment periods $i\left(i \in \mathcal{I}_{0}\right)$ ancestor to operational year $i(i \in \mathcal{I})$

$\mathcal{L} \quad$ set of transmission branches

$\mathcal{P} \quad$ set of technologies

$\mathcal{Z} \quad$ set of all locations, including platforms, $\mathrm{OEH}$, and onshore buses $\left(\mathcal{Z}^{P} \cup \mathcal{Z}^{H} \cup \mathcal{Z}^{O}\right)$

\section{Operation related sets}

$\mathcal{B}^{E} \quad$ set of electric boilers

$\mathcal{C}^{\operatorname{Exp}} \quad$ set of natural gas export compressors

$\mathcal{C}^{\operatorname{Inj}} \quad$ set of natural gas injection compressors

$\mathcal{D}^{\text {Sep }} \quad$ set of separators

$\mathcal{E} \quad$ set of electrolysers

$\mathcal{F} \quad$ set of fuel cells

$\mathcal{G} \quad$ set of gas turbines

$\mathcal{L} \quad$ set of subsea cables

$\mathcal{P}^{O} \quad$ set of oil export pumps 


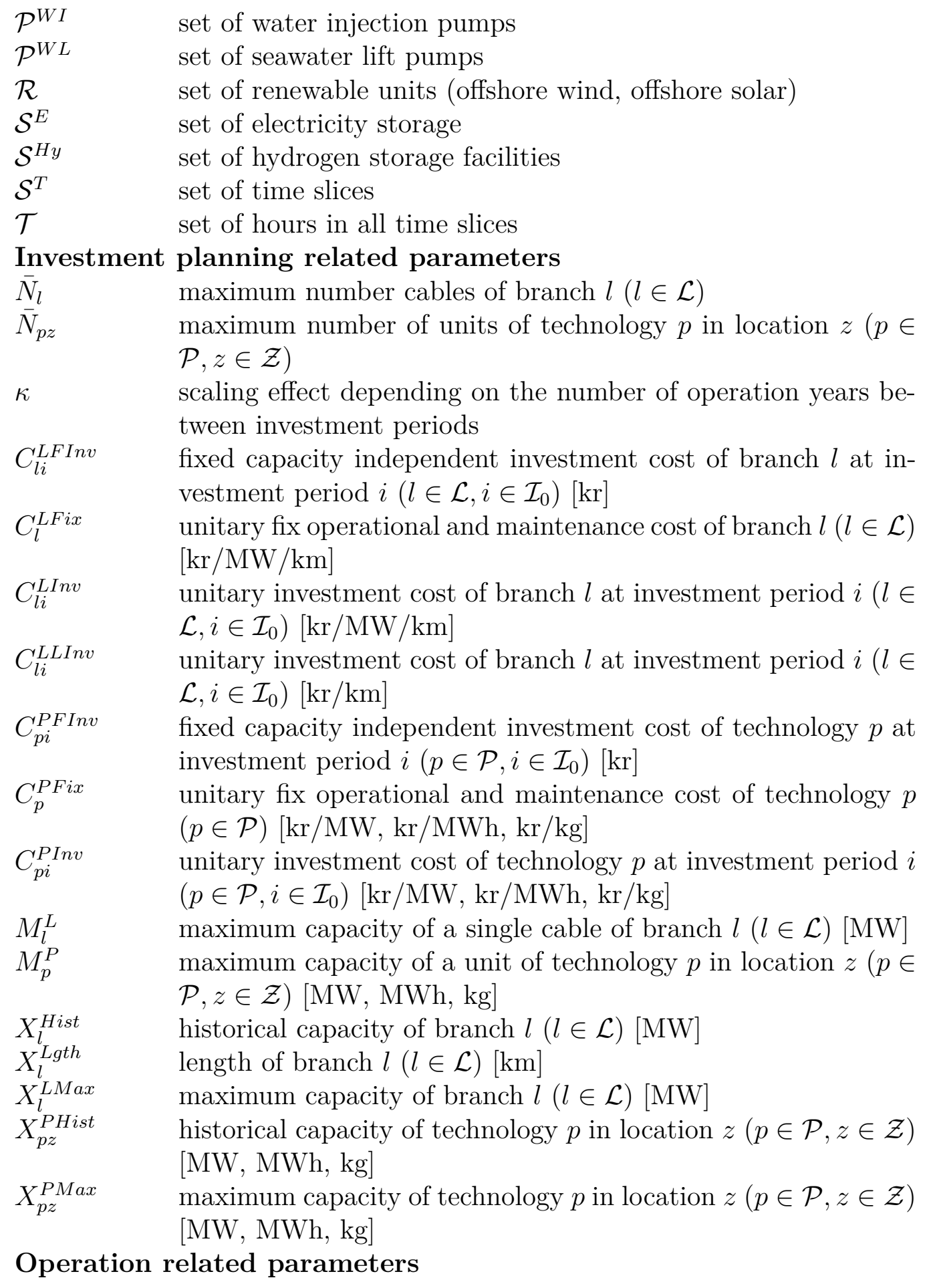


$\alpha$

$\eta^{C}$

$\eta_{b}^{E B}$

$\eta^{E F}$

$\eta^{E S}$

$\eta_{f}^{F}$

$\eta_{g}^{G h}$

$\eta_{g}^{G}$

$\eta_{l}^{L}$

$\eta_{s}^{S E}$

$\gamma_{c}$

$\kappa_{p}^{P O}$

$\kappa_{p}^{P W I}$

$\kappa_{p}^{P W L}$

$\mu^{E}$

$\rho_{d}^{S E P}$

$\sigma_{z}^{R e s}$

$\theta^{\stackrel{z}{H} y}$

$A_{z l}^{E}$

$\mathrm{C}^{\mathrm{CO}_{2}}$

$C^{\text {GShed }}$

$C^{H L S h e d}$

$C^{\text {LShed }}$

$\mathrm{C}_{z t}^{Z O}$

$C_{g}^{G}$

$C_{g}^{F u e l}$

$E_{g}^{\text {Fuel }}$

$F_{f}^{F R}$

$G_{g}^{G R}$ polytropic exponent of compressor

efficiency of compressor

efficiency of electric boiler $\left(b \in \mathcal{B}^{E}\right)$

conversion factor of electrolyser to inject hydrogen directly to fuel cell $[\mathrm{MWh} / \mathrm{kg}]$

conversion factor of electrolyser to inject hydrogen to the storage facility $[\mathrm{MWh} / \mathrm{kg}]$

efficiency of fuel cell $f(f \in \mathcal{F})$

heat recovery efficiency of gas turbine $g(g \in \mathcal{G})$

efficiency of gas turbine $g(g \in \mathcal{G})$

efficiency of transmission line $l(l \in \mathcal{L})$

efficiency of electricity store $s\left(s \in \mathcal{S}^{E}\right)$

compression ratio of compressor $c\left(c \in \mathcal{C}^{I n j} \cup \mathcal{C}^{E x p}\right)$

electricity demand as fraction of amount of oil pumped by pump $p\left(p \in \mathcal{P}^{O}\right)[\mathrm{MW} / \mathrm{kg}]$

electricity demand as fraction of amount of water injected by pump $p\left(p \in \mathcal{P}^{W I}\right)[\mathrm{MW} / \mathrm{kg}]$

electricity demand as fraction of amount of water lifted by pump $p\left(p \in \mathcal{P}^{W L}\right)[\mathrm{MW} / \mathrm{kg}]$

yearly $\mathrm{CO}_{2}$ emission limit $(\mathrm{t})$

heat demand as fraction of amount of oil heated during separation $\left(d \in D^{S e p}\right)[\mathrm{MW} / \mathrm{kg}]$

spinning reserve factor on platform $z\left(z \in \mathcal{Z}^{P}\right)$

hydrogen energy content $[\mathrm{MWh} / \mathrm{kg}]$

elements of bus/line incidence matrix $A^{E}(z \in \mathcal{Z}, l \in \mathcal{L})$

$\mathrm{CO}_{2} \operatorname{tax}(\mathrm{kr} / \mathrm{t})$

generation shed cost $[\mathrm{kr} / \mathrm{MW}]$

heat load shed penalty cost [kr/MW]

load shed penalty cost $[\mathrm{kr} / \mathrm{MW}]$

electricity price in onshore bus $z$ in period $t\left(z \in \mathcal{Z}^{O}, t \in \mathcal{T}\right)$ $[\mathrm{kr} / \mathrm{MW}]$

variable operational cost of generating $1 \mathrm{MW}$ power from gas turbine $g(g \in \mathcal{G})[\mathrm{kr} / \mathrm{MW}]$

fuel cost of gas turbine $g$ burning fuel with energy content 1 MWh $(g \in \mathcal{G})[\mathrm{kr} / \mathrm{MWh}]$

emission of $\mathrm{CO}_{2}$ of gas turbine $g$ burning fuel $(g \in \mathcal{G})$ [t/MWh]

ramping limit of fuel cell $f(f \in \mathcal{F})[\mathrm{MW} / \mathrm{MW}]$

ramping limit of gas turbine $g(g \in \mathcal{G})[\mathrm{MW} / \mathrm{MW}]$ 
$H_{t}$

$H_{s}^{S E}$

$P_{c z}^{a c c C E x p}$

$P_{c z}^{a c c C I n j}$

$P_{d z}^{a c c S E P}$

$P_{g z}^{a c c G}$

$P_{p z}^{a c c P O}$

$p z$
$P_{p z}^{a c c W I}$

$P_{p z}^{a c c W L}$

$R_{r t}^{R}$

$V_{z t}^{O D}$

$V_{z t}^{W B}$

$V_{z t}^{W I}$

$V_{z t}^{W L}$

$V_{z t}^{N G E}$

$V_{z t}^{N G I}$

$W_{s}^{S}$

$\gamma_{l i}^{L I n s t}$

$\gamma_{p z i}^{P I n s t}$

$x_{l i}^{L I n s t}$

$x_{l i}^{P A c c}$

$x_{p z i}^{P A c c}$ number of hour(s) in one operational period $t$ power ratio of electricity store $s\left(s \in \mathcal{S}^{E}\right)$ [MW/MWh]

capacity of compressor $c$ for exporting on platform $z\left(c \in \mathcal{C}^{\operatorname{Exp}}\right.$, $\left.z \in \mathcal{Z}^{P}\right)[\mathrm{MW}]$

capacity of compressor $c$ for injection on platform $z\left(c \in \mathcal{C}^{I n j}\right.$, $\left.z \in \mathcal{Z}^{P}\right)[\mathrm{MW}]$

capacity of separator $d^{s e p}$ on platform $z\left(d \in \mathcal{D}^{S e p}, z \in \mathcal{Z}^{P}\right)$ $[\mathrm{MW}]$

accumulated capacity of gas turbine $g$ on platform $z(g \in \mathcal{G}, z \in$ $\left.\mathcal{Z}^{P}\right)[\mathrm{MW}]$

capacity of oil pump $p$ on platform $z\left(p \in \mathcal{P}^{O}, z \in \mathcal{Z}^{P}\right)[\mathrm{MW}]$

capacity of water injection pump $p$ on platform $z\left(p \in \mathcal{P}^{W L}\right.$, $\left.z \in \mathcal{Z}^{P}\right)[\mathrm{MW}]$

capacity of water lift pump $p$ on platform $z\left(p \in \mathcal{P}^{W I}, z \in \mathcal{Z}^{P}\right)$ $[\mathrm{MW}]$

renewable generation profile of renewable unit $r$ in period $t$ $(r \in \mathcal{R}, t \in \mathcal{T})$

oil exporting level on platform $z$ in period $t\left(z \in \mathcal{Z}^{P}, t \in \mathcal{T}\right)$ [kg]

produced water on platform $z$ in period $t\left(z \in \mathcal{Z}^{P}, t \in \mathcal{T}\right)[\mathrm{kg}]$ water injection level on platform $z$ in period $t\left(z \in \mathcal{Z}^{P}, t \in \mathcal{T}\right)$ $[\mathrm{kg}]$

water lift level on platform $z$ in period $t\left(z \in \mathcal{Z}^{P}, t \in \mathcal{T}\right)[\mathrm{kg}]$ natural gas exporting level on platform $z$ in period $t(z \in$ $\left.\mathcal{Z}^{P}, t \in \mathcal{T}\right)[\mathrm{kg}]$

natural gas injection level on platform $z$ in period $t\left(z \in \mathcal{Z}^{P}, t \in\right.$ $\mathcal{T})[\mathrm{kg}]$ weight of time slice $s$

\section{planning related variables}

number of newly installed cables in branch $l$ at investment period $i_{0}\left(l \in \mathcal{L}, i \in \mathcal{I}_{0}\right)$

number of newly installed device $p$ in location $z$ at investment period $i_{0}\left(p \in \mathcal{P}, z \in \mathcal{Z}, i \in \mathcal{I}_{0}\right)$

newly installed capacity of branch $l$ at investment period $i_{0}$ $\left(l \in \mathcal{L}, i \in \mathcal{I}_{0}\right)[\mathrm{MW}]$

accumulated capacity of branch $l$ in operational year $i(l \in$ $\mathcal{L}, i \in \mathcal{I})[\mathrm{MW}]$

accumulated capacity of device $p$ in location $z$ in operational 
year $i(p \in \mathcal{P}, z \in \mathcal{Z}, i \in \mathcal{I})[\mathrm{MW}, \mathrm{MWh}, \mathrm{kg}]$

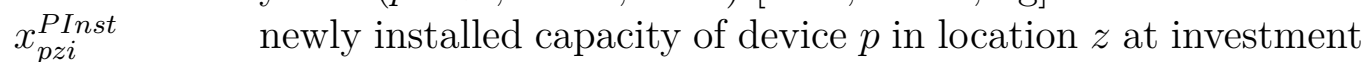
period $i_{0}\left(p \in \mathcal{P}, z \in \mathcal{Z}, i \in \mathcal{I}_{0}\right)[\mathrm{MW}, \mathrm{MWh}, \mathrm{kg}]$

\section{Operation related variables}

$p_{b z}^{a c c E B}$

$p_{e z t}^{E}$

$p_{f z t}^{F}$

$p_{b z t}^{E B}$

$p_{c z t}^{C E x p}$

$p_{c z t}^{C I n j}$

$p_{d z t}^{H S E P}$

$p_{e z}^{a c c E}$

$p_{f z}^{a c c F}$

$p_{g z t}^{G}$

$p_{g z t}^{R e s G}$

$p_{l t}^{L}$

$p_{l}^{a c c L}$

$p_{p z t}^{P O}$

$p_{p z t}^{P W I}$

$p_{p z t}^{P W L}$

$p_{r z t}^{R}$

$p_{s z t}^{R e s S E}$ accumulated capacity of electric boiler $b$ on platform $z(b \in$ $\mathcal{B}^{E}, z \in \mathcal{Z}^{P}$ ) [MW]

power input of electrolyser $e$ on $\mathrm{OEH} z$ in period $t(e \in \mathcal{E}, z \in$ $\left.\mathcal{Z}^{H}, t \in \mathcal{T}\right)[\mathrm{MW}]$

power output of fuel cell $f$ on $\mathrm{OEH} z$ in period $t(f \in \mathcal{F}, z \in$ $\left.\mathcal{Z}^{H}, t \in \mathcal{T}\right)[\mathrm{MW}]$

electricity input to electric boiler $b$ on platform $z$ in period $t$ $\left(b \in \mathcal{B}^{E}, z \in \mathcal{Z}^{P}, t \in \mathcal{T}\right)[\mathrm{MW}]$

power demand of the compressor $c$ on platform $z$ in period $t$ $\left(c \in \mathcal{C}^{E x p}, z \in \mathcal{Z}^{P}, t \in \mathcal{T}\right)[\mathrm{MW}]$

power demand of the compressor $c$ on platform $z$ in period $t$ $\left(c \in \mathcal{C}^{\text {Inj }}, z \in \mathcal{Z}^{P}, t \in \mathcal{T}\right)[\mathrm{MW}]$

heat demand of separator $d$ on platform $z$ in period $t(d \in$ $\left.\mathcal{D}^{S e p}, z \in \mathcal{Z}^{P}, t \in \mathcal{T}\right)[\mathrm{MW} / \mathrm{kg}]$

accumulated capacity of electrolyser $e$ on platform $z(e \in \mathcal{E}, z \in$ $\left.\mathcal{Z}^{H}, t \in \mathcal{T}\right)[\mathrm{MW}]$

accumulated capacity of fuel cell $f$ on platform $z(f \in \mathcal{F}, z \in$ $\left.\mathcal{Z}^{H}, t \in \mathcal{T}\right)[\mathrm{MW}]$

power output of gas turbine $g$ on platform $z$ in period $t(g \in$ $\left.\mathcal{G}, z \in \mathcal{Z}^{P}, t \in \mathcal{T}\right)[\mathrm{MW}]$

power reserved of gas turbine $g$ for spinning reserve requirement on platform $z^{p}$ in period $t\left(g \in \mathcal{G}, z \in \mathcal{Z}^{P}, t \in \mathcal{T}\right)[\mathrm{MW}]$

power flow in line $l$ in period $t(l \in \mathcal{L}, t \in \mathcal{T})[\mathrm{MW}]$

accumulated capacity of line $l(l \in \mathcal{L})[\mathrm{MW}]$

oil exporting pump power $p$ power consumption on platform $z$ in period $t\left(p \in \mathcal{P}^{O}, z \in \mathcal{Z}^{P}, t \in \mathcal{T}\right)[\mathrm{MW}]$

water injection pump $p$ power consumption on platform $z$ in period $t\left(p \in \mathcal{P}^{W I}, z \in \mathcal{Z}^{P}, t \in \mathcal{T}\right)[\mathrm{MW}]$

seawater lift pump $p$ power consumption on platform $z$ in period $t\left(p \in \mathcal{P}^{W L}, z \in \mathcal{Z}^{P}, t \in \mathcal{T}\right)[\mathrm{MW}]$

power output of renewable unit $r$ near energy hub $z$ in period $t\left(r \in \mathcal{R}, z \in \mathcal{Z}^{H}, t \in \mathcal{T}\right)[\mathrm{MW}]$

power reserved in electricity store $s$ for spinning reserve requirement on platform $z$ in period $t\left(s \in \mathcal{S}^{E}, z \in \mathcal{Z}^{P}, t \in \mathcal{T}\right)$ 
[MW]

$p_{s z t}^{S E+(-)} \quad$ charge (discharge) power of electricity store $s$ on platform $z$ in period $t\left(s \in \mathcal{S}^{E}, z \in \mathcal{Z}^{P}, t \in \mathcal{T}\right)[\mathrm{MW}]$

$p_{z^{\circ}}^{a c Z O} \quad$ accumulated capacity of shore bus $z\left(z \in \mathcal{Z}^{O}\right)[\mathrm{MW}]$

$p_{z t}^{D} \quad$ total power demand on platform $z$ in period $t\left(z \in \mathcal{Z}^{P}\right)$ [MW]

$p_{z t}^{G \text { Shed }} \quad$ electricity generation shed at $z$ in period $t(z \in \mathcal{Z}, t \in \mathcal{T})[\mathrm{MW}]$

$p_{z t}^{H G S h e d} \quad$ heat generation shed on platform $z$ in period $t\left(z \in \mathcal{Z}^{P}, t \in \mathcal{T}\right)$ [MW]

$p_{z t}^{H L \text { Shed }} \quad$ heat load shed on platform $z$ in period $t\left(z \in \mathcal{Z}^{P}, t \in \mathcal{T}\right)[\mathrm{MW}]$ $p_{z t}^{L S h e d} \quad$ electricity load shed on platform $z$ in period $t\left(z \in \mathcal{Z}^{P}, t \in \mathcal{T}\right)$ [MW]

$p_{z t}^{Z O} \quad$ power supply from onshore bus $z$ in period $t\left(z \in \mathcal{Z}^{O}, t \in \mathcal{T}\right)$ [MW]

$q_{s z t}^{S E} \quad$ energy level of electricity store $s$ on platform $z$ at the start of period $t\left(s \in \mathcal{S}^{E}, z \in \mathcal{Z}^{P}, t \in \mathcal{T}\right)[\mathrm{MWh}]$

$q_{s z}^{a c c S E} \quad$ accumulated storage capacity of electricity store $s$ on platform $z\left(s \in \mathcal{S}^{E}, z \in \mathcal{Z}^{P}\right)[\mathrm{MWh}]$

$v_{s z t}^{S H y+(-)} \quad$ injection (withdraw) of hydrogen to (from) hydrogen storage $s$ on $\mathrm{OEH} z$ in period $t\left(s \in \mathcal{S}^{H y}, z \in \mathcal{Z}^{H}, t \in \mathcal{T}\right)[\mathrm{kg}]$

$v_{s z t}^{S H y} \quad$ storage level of hydrogen storage $s$ on OEH $z$ in period $t(s \in$ $\left.\mathcal{S}^{H y}, z \in \mathcal{Z}^{H}, t \in \mathcal{T}\right)[\mathrm{kg}]$

$v_{s z}^{a c c S H y} \quad$ accumulated storage capacity of hydrogen store $s$ on platform $z\left(s \in \mathcal{S}^{H y}, z \in \mathcal{Z}^{H}, t \in \mathcal{T}\right)[\mathrm{kg}]$

Function

$g(\cdot, \cdot) \quad$ operational cost in operational year $i(\mathrm{kr})$

\section{Appendix B. Complete operational constraints}

$$
\begin{gathered}
g(x, c)=\sum_{s \in \mathcal{S}^{T}} W_{s}^{S}\left(\sum _ { t \in \mathcal { T } } H _ { t } \left(\sum _ { z \in \mathcal { Z } ^ { P } } \left(\sum_{g \in \mathcal{G}}\left(C_{g}^{G}+\frac{C_{g}^{F u e l}+C^{\mathrm{CO}_{2}} E_{g}^{F u e l}}{\eta_{g}^{G}}\right) p_{g z t}^{G}+\right.\right.\right. \\
\left.\left.\left.C^{L S h e d} p_{z t}^{L S h e d}+C^{H L S h e d} p_{z t}^{H L S h e d}\right)+\sum_{z \in \mathcal{Z}^{O}} C_{z t}^{Z O} p_{z t}^{Z O}\right)\right) \\
0 \leq p_{g z t}^{G}+p_{g z t}^{R e s G} \leq P_{g z}^{a c c G}, \quad g \in \mathcal{G}, z \in \mathcal{Z}^{P}, t \in \mathcal{T} \\
-P_{g z}^{a c c G} G_{g}^{G R} \leq p_{g z t}^{G}+p_{g z t}^{\text {ResG }}
\end{gathered}
$$




$$
\begin{aligned}
& -p_{g z(t-1)}^{G}-p_{g z(t-1)}^{R e s G} \leq P_{g z}^{a c c G} G_{g}^{G R}, \quad g \in \mathcal{G}, z \in \mathcal{Z}^{P}, t \in \mathcal{T} \\
& p_{r z t}^{R}=R_{r z t}^{R} p_{r z}^{a c c R}, \quad r \in \mathcal{R}, z \in \mathcal{Z}^{H}, t \in \mathcal{T} \\
& 0 \leq p_{z t}^{Z O} \leq p_{z}^{a c c Z O}, \quad z \in \mathcal{Z}^{O}, t \in \mathcal{T} \\
& q_{s z(t+1)}^{S E}=q_{s z t}^{S E}+H_{t}\left(\eta_{s}^{S E} p_{s z t}^{S E+}-p_{s z t}^{S E-}\right), s \in \mathcal{S}^{E}, z \in \mathcal{Z}^{P}, t \in \mathcal{T} \\
& H_{t}\left(p_{s z t}^{\text {ResSE }}+p_{s z t}^{S E-}\right) \leq q_{s z t}^{S E}, \quad s \in \mathcal{S}^{E}, z \in \mathcal{Z}^{P}, t \in \mathcal{T} \\
& 0 \leq p_{s z t}^{S E+} \leq H_{s}^{S E} q_{s z}^{a c c S E}, \quad s \in \mathcal{S}^{E}, z \in \mathcal{Z}^{P}, t \in \mathcal{T} \\
& 0 \leq p_{s z t}^{S E-}+p_{s z t}^{R e s S E} \leq H_{s}^{S E} q_{s z}^{a c c S E}, \quad s \in \mathcal{S}^{E}, z \in \mathcal{Z}^{P}, t \in \mathcal{T} \\
& 0 \leq q_{s z t}^{S E} \leq q_{s z}^{a c c S E}, \quad s \in \mathcal{S}^{E}, z \in \mathcal{Z}^{P}, t \in \mathcal{T} \\
& \sum_{g \in \mathcal{G}} p_{g z t}^{G}+\sum_{l \in \mathcal{L}} A_{z l}^{E} \eta_{l}^{L} p_{l t}^{L} \\
& +\sum_{s \in \mathcal{S}^{E}} p_{s z t}^{S E-}+p_{z t}^{L S h e d}= \\
& \mu^{D} p_{z t}^{D}+\sum_{s \in \mathcal{S}^{\mathcal{E}}} p_{s z t}^{S E+}+p_{z t}^{G S h e d}, \quad z \in \mathcal{Z}^{P}, t \in \mathcal{T} \\
& \sum_{r \in \mathcal{R}} p_{r z t}^{R}+\sum_{l \in \mathcal{L}} A_{z l}^{E} \eta_{l}^{L} p_{l t}^{L}+\sum_{f \in \mathcal{F}} p_{f z t}^{F}= \\
& p_{z t}^{G S h e d}+\sum_{e \in \mathcal{E}} p_{e z t}^{E}, \quad z \in \mathcal{Z}^{H}, t \in \mathcal{T} \\
& p_{z t}^{Z O}+\sum_{l \in \mathcal{L}} A_{z l}^{E} \eta_{l}^{L} p_{l t}^{L}=p_{z t}^{G S h e d}, \quad z \in \mathcal{Z}^{O}, t \in \mathcal{T} \\
& -p_{l}^{a c c L} \leq p_{l t}^{L} \leq p_{l}^{a c c L}, \quad l \in \mathcal{L}, t \in \mathcal{T} \\
& \sigma^{\text {Res }} p_{z t}^{D} \leq \sum_{g \in \mathcal{G}} p_{g z t}^{\text {Res } G}+\sum_{s \in \mathcal{S}^{E}} p_{s z t}^{\text {Res } S E}, \quad z \in \mathcal{Z}^{P}, t \in \mathcal{T} \\
& p_{z t}^{D}=\sum_{b \in \mathcal{B}^{E}} p_{b z t}^{E B}+\sum_{c \in \mathcal{C}^{E x p}} p_{c z t}^{C E x p} \\
& +\sum_{c \in \mathcal{C}^{I n j}} p_{c z t}^{C I n j}+\sum_{p \in \mathcal{P}^{O}} p_{p z t}^{P O} \\
& +\sum_{p \in \mathcal{P} W I} p_{p z t}^{P W I}+\sum_{p \in \mathcal{P}^{W L}} p_{p z t}^{P W L}, \quad z \in \mathcal{Z}^{P}, t \in \mathcal{T} \\
& \sum_{s \in \mathcal{S}^{T}} W_{s}^{S} \sum_{z \in \mathcal{Z}^{P}} \sum_{t \in \mathcal{T}} \sum_{g \in \mathcal{G}} \frac{E_{g}^{F u e l} p_{g z t}^{G} H_{t}}{\eta_{g}^{G}} \leq \mu^{E}, \\
& 0 \leq p_{b z t}^{E B} \leq p_{b z}^{a c c E B} \\
& b \in \mathcal{B}^{E}, z \in \mathcal{Z}^{P}, t \in \mathcal{T} \\
& 0 \leq p_{d z t}^{H S E P} \leq P_{d z}^{a c c S E P}, \quad d \in \mathcal{D}^{S e p}, z \in \mathcal{Z}^{P}, t \in \mathcal{T}
\end{aligned}
$$




$$
\begin{array}{ll}
\sum_{g \in \mathcal{G}} \eta_{g}^{G h} p_{g z t}^{G}+\sum_{b \in \mathcal{B}^{E}} \eta_{b}^{E b} p_{b z t}^{E B}+p_{z t}^{H L S h e d} & \\
\quad=\sum_{d \in \mathcal{D}^{S e p}} p_{d z t}^{H S E P}+p_{z t}^{H G S h e d}, & \\
p_{d z t}^{H S E P}=\rho_{d}^{S E P} V_{z t}^{O D}, & d \in \mathcal{D}^{S e p}, z \in \mathcal{Z}^{P}, t \in \mathcal{T} \\
0 \leq p_{c z t}^{C E x p} \leq P_{c z}^{a c c C E x p}, t \in \mathcal{T} \\
0 \leq p_{c z t}^{C I n j} \leq P_{c z}^{a c c C I n j}, & c \in \mathcal{C}^{E x p}, z \in \mathcal{Z}^{P}, t \in \mathcal{T} \\
p_{c z t}^{C I n j}=\frac{V_{z t}^{N G I}}{\eta^{C} H \rho}\left[\left(\gamma_{c}\right)^{\frac{\alpha-1}{\alpha}}-1\right], & c \in \mathcal{C}^{I n j}, z \in \mathcal{Z}^{P}, t \in \mathcal{T} \\
p_{c z t}^{C E x p}=\frac{V_{z t}^{N G E}}{\eta^{C} H \rho}\left[\left(\gamma_{c}\right)^{\frac{\alpha-1}{\alpha}}-1\right], & c \in \mathcal{Z}^{P}, t \in \mathcal{T} \\
0 \leq p_{p z t}^{P O} \leq P_{p z}^{a c c P O}, & p \in \mathcal{P}^{O}, z \in \mathcal{Z}^{P}, t \in \mathcal{T} \\
p_{p z t}^{P O}=\kappa_{p}^{P O} V_{z t}^{O D}, & p \in \mathcal{P}^{O}, z \in \mathcal{Z}^{P}, t \in \mathcal{T} \\
0 \leq p_{p z t}^{P W} \leq P_{p z}^{a c c P W I}, & p \in \mathcal{P}^{W I}, z \in \mathcal{Z}^{P}, t \in \mathcal{T} \\
0 \leq p_{p z t}^{P W L} \leq P_{p z}^{a c c P W L}, & p \in \mathcal{P}^{W L}, z \in \mathcal{Z}^{P}, t \in \mathcal{T} \\
p_{p z t}^{P W I}=\kappa_{p}^{P W I} V_{z t}^{W I}, & p \in \mathcal{P}^{W I}, z \in \mathcal{Z}^{P}, t \in \mathcal{T} \\
p_{p z t}^{P W L}=\kappa_{p}^{P W L} V_{z t}^{W L}, & p \in \mathcal{P}^{W L}, z \in \mathcal{Z}^{P}, t \in \mathcal{T}
\end{array}
$$

$$
\begin{array}{lr}
0 \leq p_{\text {ezt }}^{E} \leq p_{\text {ez }}^{a c c E}, & e \in \mathcal{E}, z \in \mathcal{Z}^{H}, t \in \mathcal{T} \\
0 \leq p_{f z t}^{F} \leq p_{f z}^{a c c F}, & f \in \mathcal{F}, z \in \mathcal{Z}^{H}, t \in \mathcal{T} \\
0 \leq v_{s z t}^{S H y} \leq v_{s z}^{a c c S H y}, & s \in \mathcal{S}^{H y}, z \in \mathcal{Z}^{H}, t \in \mathcal{T} \\
\left|p_{f z t}^{F}-p_{f z(t-1)}^{F}\right| \leq F_{f}^{F R} p_{f z}^{a c c F}, & f \in \mathcal{F}, z \in \mathcal{Z}^{H}, t \in \mathcal{T} \\
v_{s z(t+1)}^{S H y}=v_{s z t}^{S H y}+v_{s z t}^{S H y+}-v_{s z t}^{S H y-}, & s \in \mathcal{S}^{H y}, z \in \mathcal{Z}^{H}, t \in \mathcal{T} \\
\eta^{E F}\left(\sum_{f \in \mathcal{F}} \frac{p_{f z t}^{F} H_{t}}{\eta_{f}^{F} \theta^{H y}}-\sum_{s \in \mathcal{S}^{H y}} v_{s z t}^{S H y-}\right)= & \\
\sum_{e \in \mathcal{E}} p_{\text {ezt }}^{E} H_{t}-\eta^{E S} \sum_{s \in \mathcal{S}^{H y}} v_{s z t}^{S H y+}, & z \in \mathcal{Z}^{H}, t \in \mathcal{T} .
\end{array}
$$

(B.1b)-(B.1q) give the electricity system constraints. (B.2a)-(B.2m) give the platform production constraints. (B.3a)-(B.3f) represent hydrogen system constraints of OEHs. All variables except for power transmission is non- 
negative. Power transmission is a free variable. All variables are also indexed by operational year $i$, and we omit them for ease of notation.

\section{Appendix C. Calculation of energy loss}

The indices, summation and multiplication of one hour are omitted.

$$
\begin{aligned}
q^{\text {Loss }}= & p^{G S h e d}+p^{H G S h e d}+\left(\frac{1}{\eta^{G}}-1-\eta^{G h}\right) p^{G}+\left(1-\eta^{S E}\right) p^{S E+} \\
& +\left(1-\eta^{S E P}\right) p^{H S E P}+\left(1-\eta^{C}\right)\left(p^{C E x p}+p^{C I n j}\right)+\left(1-\eta^{P O}\right) p^{O} \\
& +\left(1-\eta^{P W L}\right) p^{P W L}+p^{E}-\theta^{H y}\left(\frac{p^{F}}{\eta^{F} \theta^{H y}}-v^{S H y-}+v^{S H y+}\right) \\
& +\left(\frac{1}{\eta^{F}}-1\right) p^{F}+\left(1-\eta^{L}\right) p^{L}+\left(1-\eta^{P W I}\right) p^{P W I}+\left(1-\eta^{E B}\right) p^{E B}
\end{aligned}
$$




\section{References}

[1] Backe, S., Ahang, M., \& Tomasgard, A. (2021). Stable stochastic capacity expansion with variable renewables: Comparing moment matching and stratified scenario generation sampling. Applied Energy, 302, 117538. doi:doi.org/10.1016/j.apenergy . 2021.117538.

[2] Birge, J. R., \& Louveaux, F. (2011). Introduction to stochastic programming. Springer Science \& Business Media. doi:10.1007/978-1-4614-0237-4.

[3] Bret-Rouzaut, N., Favennec, J., Babusiaux, D., \& Pearse, J. (2011). Oil and gas exploration and production: reserves, costs, contracts. doi:10.5860/choice.424042.

[4] Danish Energy Agency (2021). Denmark's Energy Islands. https://ens .dk/en/ourresponsibilities/wind-power/energy-islands/denmarks-energy-islands. [accessed September 2021].

[5] Dincer, I., Cozzani, V., \& Crivellari, A. (2021). Hybrid Energy Systems for Offshore Applications. Hybrid Energy Systems. Elsevier. doi:doi.org/10.1016/C2020-002794-5.

[6] Dunning, I., Huchette, J., \& Lubin, M. (2017). Jump: A modeling language for mathematical optimization. SIAM Review, 59, 295-320. doi:10.1137/15M1020575.

[7] Equinor (2020). Hywind tampen: the world's first renewable power for offshore oil and gas. https://www.equinor.com/en/what-we-do/hywind-tampen.html. [accessed May 2021].

[8] Equinor (2021). Reducing our greenhouse gas emissions. https://www. equinor.com/ en/sustainability/our-approach/reducing-ghg-emissions.html. [accessed July 2021].

[9] European Commission (2020). Boosting offshore renewable energy for a climate neutral europe. https://ec.europa.eu/commission/presscorner/detail/en/IP_20_ 2096. [accessed July 2021].

[10] European Commission (2020). A european green deal. https://ec.europa.eu/ info/strategy/priorities-2019-2024/european-green-deal_en. [accessed June 2021].

[11] Foss, B., Knudsen, B. R., \& Grimstad, B. (2018). Petroleum production optimization - a static or dynamic problem? Computers 8 Chemical Engineering, 114, 245-253. doi:doi.org/10.1016/j. compchemeng.2017.10.009.

[12] Gea Bermúdez, J., Koivisto, M. J., Kitzing, L., Ramos, A., \& Pedersen, R. B. B. (2021). Going offshore or not: Where to generate hydrogen in future integrated energy systems? TechRxiv, . doi:doi.org/10.36227/techrxiv.14806647.v2.

[13] Geidl, M., Koeppel, G., Favre-Perrod, P., Klockl, B., Andersson, G., \& Frohlich, K. (2007). Energy hubs for the future. IEEE Power and Energy Magazine, 5, 24-30. doi:10.1109/MPAE. 2007.264850.

[14] Gondal, I. A. (2019). Offshore renewable energy resources and their potential in a green hydrogen supply chain through power-to-gas. Sustainable Energy Fuels, 3, 1468-1489. doi:10.1039/C8SE00544C.

[15] Gorenstein Dedecca, J., \& Hakvoort, R. A. (2016). A review of the north seas offshore grid modeling: Current and future research. Renewable and Sustainable Energy Reviews, 60,129-143. doi:doi.org/10.1016/j.rser.2016.01.112. 
[16] Gunnerud, V., Foss, B., McKinnon, K., \& Nygreen, B. (2012). Oil production optimization solved by piecewise linearization in a branch \& price framework. Computers E3 Operations Research, 39, 2469-2477. doi:doi.org/10.1016/j.cor.2011.12.013.

[17] Gupta, V., \& Grossmann, I. E. (2012). An efficient multiperiod minlp model for optimal planning of offshore oil and gas field infrastructure. Industrial \& Engineering Chemistry Research, 51, 6823-6840. doi:10.1021/ie202959w.

[18] Gurobi Optimization, LLC (2021). Gurobi Optimizer Reference Manual. "https: //www.gurobi.com". [accessed May 2021].

[19] He, W., Jacobsen, G., Anderson, T., Olsen, F., Hanson, T. D., Korpås, M., Toftevaag, T., Eek, J., Uhlen, K., \& Johansson, E. (2010). The potential of integrating wind power with offshore oil and gas platforms. Wind Engineering, 34, 125-137. doi:10. 1260/0309-524X.34.2.125.

[20] Kaut, M., Midthun, K. T., Werner, A. S., Tomasgard, A., Hellemo, L., \& Fodstad, M. (2014). Multi-horizon stochastic programming. Computational Management Science, 11, 179-193. doi:10.1007/s10287-013-0182-6. Special Issue: Computational Techniques in Management Science.

[21] Kitzing, L., \& González, M. G. (2020). Market arrangements for offshore wind energy networks. Technical Report DTU. URL: https: //backend.orbit.dtu.dk/ws/portalfiles/portal/222047192/NSON_DK_WP5_ offshore_market_arrangements_final.pdf.

[22] Lara, C. L., Mallapragada, D. S., Papageorgiou, D. J., Venkatesh, A., \& Grossmann, I. E. (2018). Deterministic electric power infrastructure planning: Mixed-integer programming model and nested decomposition algorithm. European Journal of Operational Research, 271, 1037-1054. doi:doi.org/10.1016/j.ejor.2018.05.039.

[23] Lindegaard, A., Aasrud, A., Andersen, A., Andresen, S. R., Asphjell, T., Backer, E. B., Birkeli, K., Ekroll, K., Frigstad, H., Gade, H., Gjerald, E., Haugland, H., Hoem, B. M., Holmengen, N., Kasin, I., Kolshus, H., Kvalevåg, M. M., Laird, H. B., Maass, C., Møyland, E., Olbergsveen, H. R., Pettersen, T. S., Ramberg, S. H., Rikheim, B., Rosland, A., Sandgrind, S., Selboe, O. K., Skogen, S. G., Weidemann, F., \& Økstad, E. (2014). Kunnskapsgrunnlag for lavutslippsutvikling. Technical Report Miljødirektoratet. URL: https://www.miljodirektoratet.no/publikasjoner/ 2014/oktober-2014/kunnskapsgrunnlag-for-lavutslippsutvikling/.

[24] Looney, B. (2020). Statistical Review of World Energy, 2020. Technical Report Bp. URL: https://www.bp.com/content/dam/bp/business-sites/en/global/ corporate/pdfs/energy-economics/statistical-review/bp-stats-review2020-full-report.pdf.

[25] Marvik, J. I., Øyslebø, E. V., \& Korpås, M. (2013). Electrification of offshore petroleum installations with offshore wind integration. Renewable Energy, 50, 558 564. doi:doi.org/10.1016/j.renene.2012.07.010.

[26] Mazzetti, M. J., Nekså, P., Walnum, H. T., \& Hemmingsen, A. K. T. (2014). EnergyEfficiency Technologies for Reduction of Offshore CO2 Emissions. Oil and Gas Facilities, 3, 89-96. doi:10.2118/169811-PA.

[27] Mazzi, N., Grothey, A., McKinnon, K., \& Sugishita, N. (2020). Benders decomposition with adaptive oracles for large scale optimization. Mathematical programming computation, 12, 1-21. doi:doi.org/10.1007/s12532-020-00197-0. 
[28] Mikkola, E., Heinonen, J., Kankainen, M., Hekkala, T., \& Kurkela, J. (2018). Multiplatform concepts for combining offshore wind energy and fish farming in freezing sea areas: Case study in the gulf of bothnia. In Proceedings of the International Conference on Offshore Mechanics and Arctic Engineering - OMAE. volume 6 . doi:10.1115/OMAE2018-77677.

[29] Ministry of Climate and Environment (2020). Norway steps up 2030 climate goal to at least $50 \%$ towards $55 \%$. https://www.regjeringen.no/en/aktuelt/norgeforsterker-klimamalet-for-2030-til-minst-50-prosent-og-opp-mot-55prosent/id2689679/. [accessed June 2021].

[30] Mohammadi, M., Noorollahi, Y., Mohammadi-ivatloo, B., Hosseinzadeh, M., Yousefi, H., \& Khorasani, S. T. (2018). Optimal management of energy hubs and smart energy hubs - a review. Renewable and Sustainable Energy Reviews, 89, 33-50. doi:doi.org/10.1016/j.rser.2018.02.035.

[31] Mohammadi, M., Noorollahi, Y., Mohammadi-ivatloo, B., \& Yousefi, H. (2017). Energy hub: From a model to a concept - a review. Renewable and Sustainable Energy Reviews, 80,1512-1527. doi:doi.org/10.1016/j.rser.2017.07.030.

[32] NEPTUNE ENERGY (2019). Poshydon pilot, dutch north sea. https:// northseawindpowerhub.eu. [accessed May 2021].

[33] Nguyen, T.-V., Jacyno, T., Breuhaus, P., Voldsund, M., \& Elmegaard, B. (2014). Thermodynamic analysis of an upstream petroleum plant operated on a mature field. Energy, 68, 454-469. doi:doi.org/10.1016/j. energy.2014.02.040.

[34] North Sea ind Power Hub Programme (2021). North Sea wind power hub. https: //northseawindpowerhub.eu. [accessed May 2021].

[35] Norwegian Oil and Gas Association (2021). Norsk olje \& gass - enviornment. https://www.norskoljeoggass.no/miljo/miljorapporter/miljorapport2021/feltspesifikke-utslippsrapporter/. [accessed May 2021].

[36] Norwegian Petroleum Directorate (2020). Improved oil recovery (ior). https://www . norskpetroleum.no/en/environment-and-technology/emissions-to-air/. [accessed May 2021].

[37] Norwegian Petroleum Directorate (2020). Norsk petroleum. https://www. norskpetroleum.no/en/. [accessed May 2021].

[38] Norwegian Petroleum Directorate (2020). Power from Land Report. Technical Report Norwegian Petroleum Directorate. URL: https://www.npd.no/fakta/ publikasjoner/rapporter/rapportarkiv/kraft-fra-land-til-norsk-sokkel/.

[39] Norwegian Petroleum Directorate (2021). The norwegian national data repository for petroleum data. https://www.npd.no/en/diskos/. [accessed June 2021].

[40] Ødegaard Ottesen, S., Tomasgard, A., \& Fleten, S.-E. (2016). Prosumer bidding and scheduling in electricity markets. Energy, 94, 828-843. doi:doi.org/10.1016/ j.energy . 2015.11.047.

[41] Ottesen, S. O., \& Tomasgard, A. (2015). A stochastic model for scheduling energy flexibility in buildings. Energy, 88, 364-376. doi:doi .org/10.1016/j . energy . 2015. 05.049.

[42] Riboldi, L., \& Nord, L. O. (2017). Concepts for lifetime efficient supply of power and heat to offshore installations in the north sea. Energy Conversion and Management, 148, 860-875. doi:doi.org/10.1016/j .enconman.2017.06.048. 
[43] Singlitico, A., Østergaard, J., \& Chatzivasileiadis, S. (2021). Onshore, offshore or inturbine electrolysis? techno-economic overview of alternative integration designs for green hydrogen production into offshore wind power hubs. Renewable and Sustainable Energy Transition, 1, 100005. doi:doi.org/10.1016/j.rset.2021.100005.

[44] Statistics Norway (2020). Electricity. https://www.ssb.no/en/energi-ogindustri/energi/statistikk/elektrisitet. [accessed May 2021].

[45] Statistics Norway (2020). Emissions to air. https://www.ssb.no/en/natur-ogmiljo/forurensning-og-klima/statistikk/utslipp-til-luft. [accessed May 2021].

[46] Svendsen, H. G. (2013). Planning tool for clustering and optimised grid connection of offshore wind farms. Energy Procedia, 35, 297 - 306. doi:doi.org/10.1016/j. egypro.2013.07.182.

[47] Svendsen, H. G., Hadiya, M., Øyslebø, E. V., \& Uhlen, K. (2011). Integration of offshore wind farm with multiple oil and gas platforms. In 2011 IEEE Trondheim PowerTech (pp. 1-3). IEEE. doi:10.1109/PTC.2011.6019309.

[48] Tarhan, B., Grossmann, I. E., \& Goel, V. (2009). Stochastic programming approach for the planning of offshore oil or gas field infrastructure under decision-dependent uncertainty. Industrial \& Engineering Chemistry Research, 48, 3078-3097. doi:10. 1021/ie8013549.

[49] The LowEmission Research Centre, SINTEF (2020). https://www.sintef.no/ projectweb/lowemission/. [accessed May 2021].

[50] Thommessen, C., Otto, M., Nigbur, F., Roes, J., \& Heinzel, A. (2021). Technoeconomic system analysis of an offshore energy hub with an outlook on electrofuel applications. Smart Energy, 3, 100027. doi:doi.org/10.1016/j . segy.2021.100027.

[51] Trötscher, T., \& Korpås, M. (2011). A framework to determine optimal offshore grid structures for wind power integration and power exchange. Wind Energy, 14, 977 992. doi:10.1002/we.461.

[52] van der Heijde, B., Vandermeulen, A., Salenbien, R., \& Helsen, L. (2019). Representative days selection for district energy system optimisation: a solar district heating system with seasonal storage. Applied Energy, 248, 79-94. doi:doi.org/10.1016/j . apenergy.2019.04.030.

[53] van der Veer, E., Sweers, B., Kawale, D., van Unen andMiralda van Schot, M., Kee, J., Howell, F., Renz, M., de Vries RoyalHaskoningDHV: Suzan Tak, E., Knoors, B., Meijer, S., Audenaert, S., van der Keere, L., de Schutter, R., Xiao, M., ter Veld, H., Rajavelu, K., \& Pernot, E. (2019). North Sea Energy Offshore Energy Islands Deliverable D3.8. Technical Report North Sea Energy. URL: https: //north-sea-energy.eu/static/0856dd12a36d1f321aaf757706bd5913/8a.FINAL-NSE3_D3.8-Final-report-on-the-techno-economic-environmentaland-legal-assessment-of-offshore-energy-islands.pdf.

[54] Voldsund, M. (2014). Exergy analysis of offshore oil and gas processing. Ph.D. thesis NTNU Trondheim. URL: https://www.semanticscholar. org/paper/Exergy-analysis-of-offshore-oil-and-gas-processingVoldsund/421648f4f7f2a645e411c968bc9561e1d34fc515.

[55] Vår-Energi (2019). Sustainability Report. https://varenergi.no/wp-content/ 
uploads/2020/06/V\%C3\%A5r-Energi-Sustainability-Report-2019.pdf. [accessed July 2021].

[56] Zare Oskouei, M., Mohammadi-Ivatloo, B., Abapour, M., Shafiee, M., \& AnvariMoghaddam, A. (2021). Techno-economic and environmental assessment of the coordinated operation of regional grid-connected energy hubs considering high penetration of wind power. Journal of Cleaner Production, 280, 124275. doi:doi.org/10.1016/ j.jclepro.2020.124275.

[57] Zhang, A., Zhang, H., Qadrdan, M., Li, X., \& Li, Q. (2017). Energy hub based electricity generation system design for an offshore platform considering co2-mitigation. Energy Procedia, 142, 3597-3602. doi:https://doi.org/10.1016/j. egypro. 2017. 12.250. Proceedings of the 9th International Conference on Applied Energy.

[58] Zhang, H. (2021). Offmod public. doi:doi.org/10.5281/zenodo. 5163724. 